Comment. Math. Helv. 75 (2000) 319-350

(C) 2000 Birkhäuser Verlag, Basel

$0010-2571 / 00 / 020319-32 \$ 1.50+0.20 / 0$

Commentarii Mathematici Helvetici

\title{
Invariant currents on limit sets
}

\author{
John Lott
}

\begin{abstract}
We relate the $L^{2}$-cohomology of a complete hyperbolic manifold to the invariant currents on its limit set.
\end{abstract}

Mathematics Subject Classification (2000). 58G25, 57M50.

Keywords. $L^{2}$-cohomology, harmonic form, hyperbolic, limit set.

\section{Introduction}

Let $M$ be a complete oriented connected $n$-dimensional hyperbolic manifold. We can write $M=H^{n} / \Gamma$, where $\Gamma$ is a torsion-free discrete subgroup of $\operatorname{Isom}^{+}\left(H^{n}\right)$, the group of orientation-preserving isometries of the hyperbolic space $H^{n}$. The action of $\Gamma$ on $H^{n}$ extends to a conformal action on $S_{\infty}^{n-1}$, the sphere at infinity. For basic notions of hyperbolic geometry, we refer to [2]. Unless otherwise indicated, we assume that $\Gamma$ is nonelementary, i.e. does not have an abelian subgroup of finite index.

A major theme in the study of hyperbolic manifolds is the relationship between the properties of $M$ and the action of $\Gamma$ on $S_{\infty}^{n-1}$. For example, let $\lambda_{0}(M) \in[0, \infty)$ be the infimum of the spectrum $\sigma(\triangle)$ of the Laplacian on $M$. Let $\Lambda \subset S_{\infty}^{n-1}$ be the limit set of $\Gamma$ and let $D(\Gamma)$ be its Hausdorff dimension. Sullivan [15] showed that if $M$ is geometrically finite then

$$
\lambda_{0}(M)= \begin{cases}(n-1)^{2} / 4 & \text { if } D(\Gamma) \leq \frac{n-1}{2} \\ D(\Gamma)(n-1-D(\Gamma)) & \text { if } D(\Gamma) \geq \frac{n-1}{2}\end{cases}
$$

Thus there is a strong relationship between the spectrum of the Laplacian, acting on functions on $M$, and the geometry of the limit set. There is also a Laplacian $\triangle_{p}$ on $p$-forms on $M$ (see, for example, [9]). The motivating question of this paper is : What, if any, is the relationship between the spectrum of $\triangle_{p}$ and the geometry of the limit set?

Research supported by NSF grant DMS-9704633. 
If $p>0$, it is clear that the infimum of the spectrum of $\triangle_{p}$ depends on more than just the limit set as a set. For example, let $M$ be a closed hyperbolic 3manifold. From Hodge theory, $0 \in \sigma\left(\triangle_{1}\right)$ if and only if the first Betti number $b_{1}(M)$ of $M$ is nonzero. There are examples with $b_{1}(M)=0$ and examples with $b_{1}(M) \neq 0$. However, in either case, $\Lambda=S_{\infty}^{2}$.

In this paper, we address the question of whether $\operatorname{Ker}\left(\triangle_{p}\right) \neq 0$ for a hyperbolic manifold $M$. We show how the answer to the question is related to the existence of $\Gamma$-invariant $p$-currents on $S_{\infty}^{n-1}$, of a certain regularity. In some sense, these currents probe the finer geometry of the limit set.

In order to state our results, let us recall the notion of harmonic extension of $p$-forms. We use the hyperbolic ball model for $H^{n}$, with boundary $S^{n-1}$. The space of $p$-hyperforms on $S^{n-1}$ is the dual space to the space of real-analytic $(n-1-p)$-forms on $S^{n-1}$. We think of a $p$-hyperform on $S^{n-1}$ as a $p$-form whose coefficient functions are hyperfunctions. A $p$-current on $S^{n-1}$ is a $p$-hyperform whose coefficient functions are distributions.

There is a Poisson transform $\Phi_{p}$ from $p$-hyperforms on $S^{n-1}$ to coclosed harmonic $p$-forms on $H^{n}$ [6]. To describe $\Phi_{p}$ in terms of visual extension, let $\omega$ be a $p$-hyperform on $S^{n-1}$. Given $x \in H^{n}$, let $S_{x}$ be the unit sphere in $T_{x} H^{n}$ and let $A_{x}: S_{x} \rightarrow S^{n-1}$ be the visual map. Given $v \in T_{x} H^{n} \cong T_{0}\left(T_{x} H^{n}\right)$, define a vector field $V$ on $S_{x}$ by saying that at $y \in S_{x}, V$ is the translation of $v$ in $T_{x} H^{n}$ from 0 to $y$, followed by orthogonal projection onto $T_{y} S_{x}$. Then for $v_{1}, \ldots v_{p} \in T_{x} H^{n}$,

$$
\left\langle\Phi_{p}(\omega), v_{1} \wedge \ldots \wedge v_{p}\right\rangle=\frac{1}{\operatorname{vol}\left(S^{n-1}\right)} \int_{S_{x}}\left\langle A_{x}^{*} \omega, V_{1} \wedge \ldots \wedge V_{p}\right\rangle d \mathrm{vol} .
$$

Equivalently, given $x \in H^{n}$ and $v \in T_{x} H^{n}$, take an upper-half-space model

$$
\left\{\left(x_{1}, \ldots, x_{n}\right) \in \mathbb{R}^{n}: x_{n}>0\right\}
$$

for $H^{n}$ in which $x=(0, \ldots, 0,1)$ and $v=c \frac{\partial}{\partial x_{n}}$ for some $c \in \mathbb{R}$. Consider the Killing vector field $c \sum_{i=1}^{n} x_{i} \frac{\partial}{\partial x_{i}}$. It restricts to a conformal vector field $W$ on $\partial H^{n}=S^{n-1}$. Then for $v_{1}, \ldots v_{p} \in T_{x} H^{n}$,

$$
\left\langle\Phi_{p}(\omega), v_{1} \wedge \ldots \wedge v_{p}\right\rangle=\frac{1}{\operatorname{vol}\left(S^{n-1}\right)} \int_{S^{n-1}}\left\langle\omega, W_{1} \wedge \ldots \wedge W_{p}\right\rangle d \mathrm{vol} .
$$

By a result of Gaillard, for $p>0, \Phi_{p}$ is an isomorphism from exact $p$-hyperforms on $S^{n-1}$ to closed and coclosed $p$-forms on $H^{n}$ [6, Théorème 2]. Following [6], we say that a $p$-form $\alpha$ on $H^{n}$ has slow growth if there are constants $a, b>0$ such that for some (or any) $m_{0} \in H^{n}$,

$$
|\alpha(m)| \leq a e^{b d\left(m_{0}, m\right)}
$$

for all $m \in H^{n}$. Then for $p>0, \Phi_{p}$ is also an isomorphism from exact $p$-currents on $S^{n-1}$ to closed and coclosed $p$-forms on $H^{n}$ of slow growth [6, Théorème 3]. 
Let $\pi: H^{n} \rightarrow H^{n} / \Gamma$ be the quotient map. Let $\Omega=S^{n-1}-\Lambda$ be the domain of discontinuity.

By Gaillard's theorem, if $p>0$ then $\Phi_{p}^{-1} \circ \pi^{*}$ induces an isomorphism between closed and coclosed $p$-forms on $H^{n} / \Gamma$, and $\Gamma$-invariant exact p-hyperforms on $S^{n-1}$. Let $\alpha$ be an $L^{2}$-harmonic $p$-form on $H^{n} / \Gamma$. By Hodge theory, $\alpha$ is closed and coclosed. Thus we can use results about the $L^{2}$-cohomology of $H^{n} / \Gamma$ to construct $\Gamma$-invariant exact $p$-hyperforms on $S^{n-1}$, and vice versa. The questions that we address are :

1. What can we say about the regularity of these hyperforms?

2. Are they supported on the limit set?

Under Hodge duality, the space of $L^{2}$-harmonic $p$-forms on $H^{n} / \Gamma$ is isomorphic to the space of $L^{2}$-harmonic $(n-p)$-forms. Without loss of generality, hereafter we assume that $p \in\left[1, \frac{n}{2}\right]$.

Theorem 1. If $n$ is even then up to a constant, $\Phi_{\frac{n}{2}}$ is an isometric isomorphism between exact $\frac{n}{2}$-forms on $S^{n-1}$ which are Sobolev $\mathrm{H}^{-\frac{1}{2}}$-regular, and $L^{2}$-harmonic $\frac{n}{2}$-forms on $H^{n}$.

From Theorem 1, we obtain that the $\frac{n}{2}$-hyperforms that we construct on $S^{n-1}$ cannot be too regular.

Corollary 1. Suppose that $\alpha$ is a nonzero $L^{2}$-harmonic $\frac{n}{2}$-form on $H^{n} / \Gamma$. If $\Gamma$ is infinite then $\Phi_{\frac{n}{2}}^{-1}\left(\pi^{*} \alpha\right)$ is not Sobolev $\mathrm{H}^{-\frac{1}{2}}$-regular.

We now give some positive regularity results. Let us recall that $\Gamma$ is said to be cocompact if $H^{n} / \Gamma$ is compact. It is said to be convex-cocompact if there is a compact subset $K$ of $H^{n} / \Gamma$ such that all nontrivial closed geodesics in $H^{n} / \Gamma$ lie in $K$. If $\Gamma$ is convex-cocompact then $H^{n} / \Gamma$ consists of $K$ along with a finite number of flaring ends attached to $K$.

Theorem 2. A. If $\Gamma$ is cocompact then for any $p \in\left[1, \frac{n}{2}\right]$, there are isomorphisms between the following vector spaces:

$V_{1}=\left\{\right.$ Harmonic $p$-forms on $\left.H^{n} / \Gamma\right\}$.

$V_{2}=\left\{\Gamma\right.$-invariant exact $p$-hyperforms on $\left.S^{n-1}\right\}$.

$V_{3}=\left\{\Gamma\right.$-invariant exact $p$-currents on $S^{n-1}$ which are Sobolev $\mathrm{H}^{-p-\epsilon}$-regular for all $\epsilon>0\}$.

$V_{4}=\mathrm{H}^{p}\left(H^{n} / \Gamma, \mathbb{R}\right)$, the p-dimensional real cohomology group of $H^{n} / \Gamma$.

$B$. If $\Gamma$ is convex-cocompact then for any $p \in\left[1, \frac{n-1}{2}\right)$, there are isomorphisms between the following vector spaces:

$V_{1}=\left\{L^{2}\right.$-harmonic p-forms on $\left.H^{n} / \Gamma\right\}$.

$V_{2}=\left\{\Gamma\right.$-invariant exact $p$-hyperforms on $S^{n-1}$ which are supported on the limit set\}. 
$V_{3}=\left\{\Gamma\right.$-invariant exact $p$-currents on $S^{n-1}$ which are supported on the limit set and which are Sobolev $\mathrm{H}^{-p-\epsilon}$-regular for all $\left.\epsilon>0\right\}$.

$V_{4}=\mathrm{H}_{c}^{p}\left(H^{n} / \Gamma, \mathbb{R}\right)$, the p-dimensional real compactly-supported cohomology group of $H^{n} / \Gamma$.

In Theorem 2, we show that the injection $V_{3} \rightarrow V_{2}$ is surjective and that $\Phi_{p}$ induces an isomorphism from $V_{2}$ to $V_{1}$. In case $\mathrm{A}$, there is an isomorphism between $V_{4}$ and $V_{1}$ from standard Hodge theory. By [12], this is also true in case B.

There are extensions of Theorem 2 to hyperbolic manifolds with vanishing injectivity radius. We state one such extension here.

Theorem 3. If $n=3$, suppose that there is a positive lower bound to the lengths of the nontrivial closed geodesics on $H^{3} / \Gamma$. Let $\alpha$ be an $L^{2}$-harmonic 1-form on $H^{3} / \Gamma$. Then for all $\epsilon>0$, the hyperform $\Phi_{1}^{-1}\left(\pi^{*} \alpha\right)$ is Sobolev $\mathrm{H}^{-1-\epsilon}$-regular.

We show that the regularity estimate in Theorem 2 is sharp in some cases. We find an interesting distinction between cocompact groups, and convex-cocompact groups which are not cocompact.

Theorem 4. A. Suppose that $\Gamma$ is cocompact. Let $\alpha$ be a nonzero harmonic 1form on $H^{n} / \Gamma$. Then $\Phi_{1}^{-1}\left(\pi^{*} \alpha\right)$ is not Sobolev $\mathrm{H}^{-1}$-regular.

B. Let $\Gamma$ be a convex-cocompact group which is not cocompact. Let $\alpha$ be an $L^{2}$ harmonic 1-form on $H^{n} / \Gamma$. Then $\Phi_{1}^{-1}\left(\pi^{*} \alpha\right)$ is Sobolev $\mathrm{H}^{-1}$-regular.

We look at what our general results become in the case of surfaces and 3manifolds. In the case of surfaces, we obtain results about the actions of Fuchsian groups on certain function spaces on $S^{1}$. Let $\mathcal{A}^{\prime}\left(S^{1}\right)$ denote the hyperfunctions on $S^{1}$ and let $\mathcal{A}_{0}^{\prime}\left(S^{1}\right)$ denote those which vanish on constant functions. Let $\mathcal{D}^{\prime}\left(S^{1}\right)$ denote the distributions on $S^{1}$ and let $\mathcal{D}_{0}^{\prime}\left(S^{1}\right)$ denote those which vanish on constant functions. Recall that a Zygmund function on $S^{1}$ is a function $f: S^{1} \rightarrow \mathbb{C}$ such that

$$
\sup _{x \in S^{1}, h \in \mathbb{R}^{+}} \frac{|f(x+h)+f(x-h)-2 f(x)|}{h}<\infty .
$$

A Zygmund function is continuous and lies in the Sobolev space $\mathrm{H}^{1-\epsilon}\left(S^{1}\right)$ for all $\epsilon>0$. Let $\mathcal{D} Z\left(S^{1}\right)$ denote the generalized functions on $S^{1}$ which are derivatives of Zygmund functions, plus constants. If $\Gamma$ is a subgroup of $\operatorname{PSL}(2, \mathbb{R})$, let $\left(\mathcal{A}_{0}^{\prime}\left(S^{1}\right)\right)^{\Gamma}$ denote the $\Gamma$-invariant subspace of $\mathcal{A}_{0}^{\prime}\left(S^{1}\right)$, and similarly for $\left(\mathcal{D}_{0}^{\prime}\left(S^{1}\right)\right)^{\Gamma}$ and $\left(\mathcal{D} Z\left(S^{1}\right) / \mathbb{C}\right)^{\Gamma}$.

Theorem 5. A. Let $\Gamma$ be a torsion-free uniform lattice in $\operatorname{Isom}^{+}\left(H^{2}\right)$, with $H^{2} / \Gamma$ a closed surface of genus $g$. Then

1. $\operatorname{dim}\left(\mathcal{A}_{0}^{\prime}\left(S^{1}\right)\right)^{\Gamma}=2 g$. 
2. $\operatorname{dim}\left(\mathcal{D}_{0}^{\prime}\left(S^{1}\right)\right)^{\Gamma}=2 g$.

3. $\operatorname{dim}\left(\mathcal{D} Z\left(S^{1}\right) / \mathbb{C}\right)^{\Gamma}=2 g$.

4. $\operatorname{dim}\left(L^{2}\left(S^{1}\right) / \mathbb{C}\right)^{\Gamma}=0$.

B. Let $\Gamma$ be a torsion-free nonuniform lattice in $\operatorname{Isom}^{+}\left(H^{2}\right)$, with $H^{2} / \Gamma$ the complement of $k$ points in a closed surface $S$ of genus $g$. Then

1. $\operatorname{dim}\left(\mathcal{A}_{0}^{\prime}\left(S^{1}\right)\right)^{\Gamma}=\infty$.

2. $\operatorname{dim}\left(\mathcal{D}_{0}^{\prime}\left(S^{1}\right)\right)^{\Gamma}=\max (2 g, 2 g+2 k-2)$.

3. $\operatorname{dim}\left(H^{-\frac{1}{2}}\left(S^{1}\right) / \mathbb{C}\right)^{\Gamma}=2 g$.

4. $\operatorname{dim}\left(\mathcal{D} Z\left(S^{1}\right) / \mathbb{C}\right)^{\Gamma}=2 g$.

5. $\operatorname{dim}\left(L^{2}\left(S^{1}\right) / \mathbb{C}\right)^{\Gamma}=0$.

Parts A.2 and B.2 of Theorem 5 are due to Haefliger and Banghe [8].

Next, we look at the case of quasi-Fuchsian 3-manifolds. We follow the philosophy of Connes and Sullivan [5, Section IV.3. $\gamma$ ]. Let $S$ be a closed oriented surface of genus $g>1$. Let $\Gamma$ be a quasi-Fuchsian subgroup of $\operatorname{Isom}^{+}\left(H^{3}\right)$ which is isomorphic to $\pi_{1}(S)$. Then $H^{3} / \Gamma$ is diffeomorphic to $\mathbb{R} \times S$ and $\mathrm{H}_{c}^{1}\left(H^{3} / \Gamma ; \mathbb{C}\right)=\mathbb{C}$. Thus there is a nonzero $L^{2}$-harmonic 1-form $\alpha$ on $H^{3} / \Gamma$.

We show that $\Phi_{1}^{-1}\left(\pi^{*} \alpha\right)$ is a $\Gamma$-invariant exact 1-current supported on the limit set $\Lambda \subset S^{2}$. The domain of discontinuity $\Omega \subset S^{2}$ is the union of two 2-disks $D_{+}$ and $D_{-}$, with $D_{+} / \Gamma$ and $D_{-} / \Gamma$ homeomorphic to $S$. Let $\chi_{D_{+}} \in L^{2}\left(S^{2}\right)$ be the characteristic function of $D_{+}$. We show that $\Phi_{1}^{-1}\left(\pi^{*} \alpha\right)$ is proportionate to the exact 1-current $d \chi_{D_{+}}$on $S^{2}$.

Let $Z: D^{2} \rightarrow D_{+}$be a uniformization of $D_{+}$. By Carathéodory's theorem, $Z$ extends to a continuous homeomorphism $\bar{Z}: \overline{D^{2}} \rightarrow \overline{D_{+}}$. The restriction of $\bar{Z}$ to $\partial \overline{D^{2}}$ gives a homeomorphism $\partial \bar{Z}: S^{1} \rightarrow \Lambda$.

The 1-current $d \chi_{D_{+}}$defines a cyclic 1-cocycle $\tau$ on the algebra $C^{1}\left(S^{2}\right)$ by

$$
\tau\left(F^{0}, F^{1}\right)=\int_{S^{2}} d \chi_{D_{+}} \wedge F^{0} d F^{1}
$$

Lemma 1. The function space $\mathrm{H}^{\frac{1}{2}}\left(S^{1}\right) \cap L^{\infty}\left(S^{1}\right)$ is a Banach algebra with the norm

$$
\|f\|=\left(\int_{\mathbb{R}^{+}} \int_{S^{1}} \frac{|f(\theta+h)-f(\theta)|^{2}}{h^{2}} d \theta d h\right)^{\frac{1}{2}}+\|f\|_{\infty} .
$$

Given $f^{0}, f^{1} \in \mathrm{H}^{\frac{1}{2}}\left(S^{1}\right) \cap L^{\infty}\left(S^{1}\right)$, let

$$
f^{i}(\theta)=\sum_{j \in \mathbb{Z}} c_{j}^{i} e^{\sqrt{-1} j \theta}
$$


be the Fourier expansion. Define a bilinear function

$$
\bar{\tau}:\left(\mathrm{H}^{\frac{1}{2}}\left(S^{1}\right) \cap L^{\infty}\left(S^{1}\right)\right) \times\left(\mathrm{H}^{\frac{1}{2}}\left(S^{1}\right) \cap L^{\infty}\left(S^{1}\right)\right) \rightarrow \mathbb{C}
$$

by

$$
\bar{\tau}\left(f^{0}, f^{1}\right)=-2 \pi i \sum_{j \in \mathbb{Z}} j c_{j}^{0} c_{-j}^{1}
$$

Then $\bar{\tau}$ is a continuous cyclic 1-cocycle on $\mathrm{H}^{\frac{1}{2}}\left(S^{1}\right) \cap L^{\infty}\left(S^{1}\right)$.

We relate the function-theoretic 1-cocycle $\bar{\tau}$ to the 1-cocycle $\tau$.

Theorem 6. Given $F^{0}, F^{1} \in C^{1}\left(S^{2}\right)$, put $f^{i}=(\partial \bar{Z})^{*} F^{i}, i \in\{1,2\}$. Then $f^{i} \in \mathrm{H}^{\frac{1}{2}}\left(S^{1}\right) \cap L^{\infty}\left(S^{1}\right)$ and

$$
\tau\left(F^{0}, F^{1}\right)=-\bar{\tau}\left(f^{0}, f^{1}\right)
$$

In Subsection 5.2 we give examples of discrete subgroups $\Gamma$ of $\operatorname{Isom}^{+}\left(H^{3}\right)$ with limit set $S^{2}$ such that for all $\epsilon>0$, the $\Gamma$-invariant subspace of $\mathrm{H}^{-\epsilon}\left(S^{2}\right) / \mathbb{C}$ is infinite-dimensional. This constrasts with the fact that from ergodicity, the $\Gamma$ invariant subspace of $L^{2}\left(S^{2}\right) / \mathbb{C}$ vanishes.

Let us remark that our results could be extended to eigenfunctions of $\triangle_{p}$ with nonzero eigenvalue. In this paper we only deal with $L^{2}$-harmonic forms since the dimension of the space of such forms can often be computed in terms of topological data, such as when $M$ is a geometrically-finite hyperbolic manifold [12].

\section{Regularity}

Let $p$ be an integer in $\left[1, \frac{n}{2}\right]$ Take coordinates $(r, \theta) \in(0,1) \times S^{n-1}$ for $H^{n}-\{0\}$, with metric

$$
d s^{2}=\frac{4\left(d r^{2}+r^{2} d \theta^{2}\right)}{\left(1-r^{2}\right)^{2}} .
$$

For $k \geq 0$, consider the hypergeometric function

$$
F_{p, k}(z)=F\left(1+p-\frac{n}{2}, 1+p+k ; 1+\frac{n}{2}+k ; z\right)
$$

Put

$$
c_{p, k}=\frac{2^{p+1}}{n} \frac{\Gamma(n-p+k) \Gamma\left(\frac{n}{2}+1\right)}{\Gamma(n-p) \Gamma\left(\frac{n}{2}+k+1\right)}=\frac{2^{p+1}}{n} \frac{(n-p)(n-p+1) \ldots(n-p+k-1)}{\left(\frac{n}{2}+1\right)\left(\frac{n}{2}+2\right) \ldots\left(\frac{n}{2}+k\right)} .
$$


Let $\left\{\alpha_{i}\right\}_{i=1}^{\infty}$ be a sequence of coclosed $(p-1)$-forms on $S^{n-1}$ such that

1. $\alpha_{i}$ is an eigenvector for the Laplacian with eigenvalue $\left(k_{i}+p\right)\left(k_{i}+n-p\right)$, $k_{i} \in \mathbb{Z} \cap[0, \infty)$.

2. $\left\{d \alpha_{i}\right\}_{i=1}^{\infty}$ is an orthonormal basis of the exact $p$-forms on $S^{n-1}$.

Then

$$
\left\|\alpha_{i}\right\|_{L^{2}}^{2}=\frac{1}{\left(k_{i}+p\right)\left(k_{i}+n-p\right)} .
$$

Given an exact $p$-hyperform $\omega$ on $S^{n-1}$, let

$$
\omega=\sum_{i=1}^{\infty} c_{i} d \alpha_{i}
$$

be its Fourier expansion. Gaillard [6, p. 599] showed that the Poisson transform of $\omega$ is

$$
\begin{aligned}
\Phi_{p}(\omega)= & \sum_{i=1}^{\infty} c_{i} \frac{\left(k_{i}+p\right)\left(k_{i}+n-p\right)}{2} c_{p, k_{i}} r^{p-1+k_{i}} \\
& \quad\left[\frac{r}{k_{i}+p} F_{p-1, k_{i}}\left(r^{2}\right) d \alpha_{i}+\left(1-r^{2}\right) F_{p, k_{i}}\left(r^{2}\right) d r \wedge \alpha_{i}\right] .
\end{aligned}
$$

Put $S^{n-1}(r)=\left\{(r, \theta): \theta \in S^{n-1}\right\} \subset H^{n}$. Given $\eta \in \Omega^{p-1}\left(S^{n-1}\right)$, we can think of $d \eta$ and $d r \wedge \eta$ as $p$-forms on $H^{n}-\{0\}$. Their pointwise norms on $S^{n-1}(r)$ are

$$
|d \eta|_{S^{n-1}(r)}=\left(\frac{1-r^{2}}{2 r}\right)^{p}|d \eta|_{S^{n-1}}
$$

and

$$
|d r \wedge \eta|_{S^{n-1}(r)}=\frac{1-r^{2}}{2}\left(\frac{1-r^{2}}{2 r}\right)^{p-1}|\eta|_{S^{n-1}}
$$

Theorem 1. If $n$ is even then up to a constant, $\Phi_{\frac{n}{2}}$ is an isometric isomorphism between exact $\frac{n}{2}$-forms on $S^{n-1}$ which are Sobolev $\mathrm{H}^{-\frac{1}{2}}$-regular, and $L^{2}$-harmonic $\frac{n}{2}$-forms on $H^{n}$.

Proof. We have

$$
\begin{gathered}
F_{\frac{n}{2}, k}(z)=F\left(1,1+\frac{n}{2}+k ; 1+\frac{n}{2}+k ; z\right)=(1-z)^{-1} \\
F_{\frac{n}{2}-1, k}(z)=F\left(0, \frac{n}{2}+k ; 1+\frac{n}{2}+k ; z\right)=1
\end{gathered}
$$

and

$$
c_{\frac{n}{2}, k}=\frac{2^{\frac{n}{2}}}{k+\frac{n}{2}} .
$$


Then

$$
\Phi_{\frac{n}{2}}(\omega)=\sum_{i=1}^{\infty} c_{i} 2^{\frac{n}{2}-1}\left[r^{\frac{n}{2}+k_{i}} d \alpha_{i}+\left(k_{i}+\frac{n}{2}\right) r^{\frac{n}{2}+k_{i}-1} d r \wedge \alpha_{i}\right] .
$$

Thus

$$
\begin{aligned}
\int_{H^{n}}\left|\Phi_{\frac{n}{2}}(\omega)\right|^{2} d \mathrm{vol}=\sum_{i=1}^{\infty}\left|c_{i}\right|^{2} 2^{n-2} \operatorname{vol}\left(S^{n-1}\right) \int_{0}^{1}\left[r^{n+2 k_{i}}\left(\frac{1-r^{2}}{2 r}\right)^{n}+\right. \\
\left.r^{n+2 k_{i}-2}\left(\frac{1-r^{2}}{2}\right)^{2}\left(\frac{1-r^{2}}{2 r}\right)^{n-2}\right]\left(\frac{2 r}{1-r^{2}}\right)^{n-1} \frac{2}{1-r^{2}} d r \\
=\sum_{i=1}^{\infty}\left|c_{i}\right|^{2} 2^{n-1} \operatorname{vol}\left(S^{n-1}\right) \int_{0}^{1} r^{2 k_{i}+n-1} d r \\
=2^{n-2} \operatorname{vol}\left(S^{n-1}\right) \sum_{i=1}^{\infty} \frac{1}{k_{i}+\frac{n}{2}}\left|c_{i}\right|^{2} .
\end{aligned}
$$

The theorem follows.

Corollary 1. Suppose that $\alpha$ is a nonzero $L^{2}$-harmonic $\frac{n}{2}$-form on $H^{n} / \Gamma$. If $\Gamma$ is infinite then $\Phi_{\frac{n}{2}}^{-1}\left(\pi^{*} \alpha\right)$ is not Sobolev $\mathrm{H}^{-\frac{1}{2}}$-regular.

Proof. If $\Phi_{\frac{n}{2}}^{-1}\left(\pi^{*} \alpha\right)$ were Sobolev $\mathrm{H}^{-\frac{1}{2}}$-regular then Theorem 1 would imply that $\pi^{*} \alpha$ is $L^{2}$, contradicting the assumption that $\Gamma$ is infinite.

The following is the main technical result of the paper.

Theorem 7. If $\omega$ is an exact p-hyperform on $S^{n-1}$ and if $\Phi_{p}(\omega)$ is $L^{\infty}$-bounded on $H^{n}$ then $\omega$ is Sobolev $\mathrm{H}^{-p-\epsilon}$-regular for all $\epsilon>0$.

Proof. By the assumptions, $\frac{1}{\operatorname{vol}\left(S^{n-1}(r)\right)} \int_{S^{n-1}(r)}\left|\Phi_{p}(\omega)\right|^{2} d \mathrm{vol}$ is uniformly bounded in $r \in(0,1)$. Thus for $\epsilon>0$,

$$
\int_{0}^{1} r\left(1-r^{2}\right)^{-1+2 \epsilon} \frac{1}{\operatorname{vol}\left(S^{n-1}(r)\right)} \int_{S^{n-1}(r)}\left|\Phi_{p}(\omega)\right|^{2} d \operatorname{vol} d r<\infty .
$$

In particular, just looking at the $d r \wedge \alpha$ component of $\Phi_{p}(\omega)$ in (2.6) gives

$$
\sum_{i=1}^{\infty}\left(k_{i}+p\right)^{2}\left(k_{i}+n-p\right)^{2} c_{p, k_{i}}^{2}\left|c_{i}\right|^{2}
$$




$$
\begin{aligned}
\int_{0}^{1} r\left(1-r^{2}\right)^{-1+2 \epsilon} r^{2 p-2+2 k_{i}}\left(1-r^{2}\right)^{2} F_{p, k_{i}}^{2}\left(r^{2}\right)\left(\frac{1-r^{2}}{2}\right)^{2}\left(\frac{1-r^{2}}{2 r}\right)^{2 p-2} \\
\quad \frac{1}{\left(k_{i}+p\right)\left(k_{i}+n-p\right)} d r<\infty
\end{aligned}
$$

or

$$
\sum_{i=1}^{\infty}\left(k_{i}+p\right)\left(k_{i}+n-p\right) c_{p, k_{i}}^{2}\left|c_{i}\right|^{2} \int_{0}^{1} z^{k_{i}}(1-z)^{2 p+1+2 \epsilon} F_{p, k_{i}}^{2}(z) d z<\infty .
$$

For the regularity question, it is the regime of large $k_{i}$ and $z$ near 1 which is relevant. Thus our main problem is to derive uniform estimates for $F_{p, k_{i}}^{2}(z)$, for large $k_{i}$ and $z$ near 1 .

Substituting $z=\frac{w-1}{w+1}$ gives

$$
\sum_{i=1}^{\infty}\left(k_{i}+p\right)\left(k_{i}+n-p\right) c_{p, k_{i}}^{2}\left|c_{i}\right|^{2} \int_{1}^{\infty}(w-1)^{k_{i}}(w+1)^{-2 p-k_{i}-3-2 \epsilon} F_{p, k_{i}}^{2}\left(\frac{w-1}{w+1}\right) d w<\infty .
$$

Restricting the summation to $k_{i}>0$, the further substitution $w=k_{i} x$ gives

$$
\begin{aligned}
& \sum_{i}\left(k_{i}+p\right)\left(k_{i}+n-p\right) c_{p, k_{i}}^{2}\left|c_{i}\right|^{2} k_{i}^{-2 p-2-2 \epsilon} \\
& \int_{k_{i}^{-1}}^{\infty} x^{-2 p-3-2 \epsilon}\left(1-\frac{1}{k_{i} x}\right)^{k_{i}}\left(1+\frac{1}{k_{i} x}\right)^{-2 p-k_{i}-3-2 \epsilon} F_{p, k_{i}}^{2}\left(\frac{k_{i} x-1}{k_{i} x+1}\right) d x<\infty .
\end{aligned}
$$

In order to estimate $F_{p, k_{i}}$, we use the transformation $[1,15.3 .4]$

$$
\begin{aligned}
F_{p, k}(z) & =F\left(1+p-\frac{n}{2}, 1+p+k ; 1+\frac{n}{2}+k ; z\right) \\
& =(1-z)^{\frac{n}{2}-p-1} F\left(1+p-\frac{n}{2}, \frac{n}{2}-p ; 1+\frac{n}{2}+k ; \frac{z}{z-1}\right) .
\end{aligned}
$$

Then

$$
F_{p, k}\left(\frac{w-1}{w+1}\right)=\left(\frac{2}{w+1}\right)^{\frac{n}{2}-p-1} F\left(1+p-\frac{n}{2}, \frac{n}{2}-p ; 1+\frac{n}{2}+k ; \frac{1}{2}-\frac{w}{2}\right) .
$$

From [11, (4) p. 246 and (15) p. 248],

$$
P_{\frac{n}{2}-p-1}^{-\frac{n}{2}-k}(w)=\frac{1}{\Gamma\left(1+\frac{n}{2}+k\right)}\left(\frac{w+1}{w-1}\right)^{-\frac{n}{4}-\frac{k}{2}} F\left(1+p-\frac{n}{2}, \frac{n}{2}-p ; 1+\frac{n}{2}+k ; \frac{1}{2}-\frac{w}{2}\right)
$$


and

$$
\int_{0}^{\infty} e^{-w t} t^{\frac{n}{2}+k-\frac{1}{2}} K_{\frac{n}{2}-p-\frac{1}{2}}(t) d t=\left(\frac{\pi}{2}\right)^{\frac{1}{2}} \frac{\Gamma(k+p+1) \Gamma(n+k-p)}{\left(w^{2}-1\right)^{\frac{n}{4}+\frac{k}{2}}} P_{\frac{n}{2}-p-1}^{-\frac{n}{2}-k}(w) .
$$

We obtain

$$
\begin{aligned}
F_{p, k}\left(\frac{w-1}{w+1}\right)= & \left(\frac{2}{\pi}\right)^{\frac{1}{2}} 2^{\frac{n}{2}-p-1} \frac{\Gamma\left(1+\frac{n}{2}+k\right)}{\Gamma(k+p+1) \Gamma(n+k-p)}(w+1)^{k+p+1} \\
& \int_{0}^{\infty} e^{-w t} t^{\frac{n}{2}+k-\frac{1}{2}} K_{\frac{n}{2}-p-\frac{1}{2}}(t) d t
\end{aligned}
$$

so

$$
\begin{aligned}
F_{p, k_{i}}\left(\frac{k_{i} x-1}{k_{i} x+1}\right)= & \left(\frac{2}{\pi}\right)^{\frac{1}{2}} 2^{\frac{n}{2}-p-1} \frac{k_{i}^{k_{i}+p+1} \Gamma\left(1+\frac{n}{2}+k_{i}\right)}{\Gamma\left(k_{i}+p+1\right) \Gamma\left(n+k_{i}-p\right)} x^{k_{i}+p+1} \\
& \left(1+\frac{1}{k_{i} x}\right)^{k_{i}+p+1} \int_{0}^{\infty} e^{-k_{i} x t} t^{\frac{n}{2}+k_{i}-\frac{1}{2}} K_{\frac{n}{2}-p-\frac{1}{2}}(t) d t
\end{aligned}
$$

(Recall that for large $t$ [1, 9.7.2 and 10.2.17],

$$
\left.K_{\frac{n}{2}-p-\frac{1}{2}}(t) \sim \sqrt{\frac{\pi}{2 t}} e^{-t} .\right)
$$

Then from $(2.20)$,

$$
\begin{aligned}
& \sum_{i}\left(k_{i}+p\right)\left(k_{i}+n-p\right) c_{p, k_{i}}^{2}\left|c_{i}\right|^{2} \frac{k_{i}^{2 k_{i}-2 \epsilon} \Gamma^{2}\left(1+\frac{n}{2}+k_{i}\right)}{\Gamma^{2}\left(k_{i}+p+1\right) \Gamma^{2}\left(n+k_{i}-p\right)} \\
& \int_{k_{i}^{-1}}^{\infty} x^{2 k_{i}-1-2 \epsilon} \int_{0}^{\infty} \int_{0}^{\infty}\left(1-\frac{1}{k_{i} x}\right)^{k_{i}}\left(1+\frac{1}{k_{i} x}\right)^{k_{i}-1-2 \epsilon} e^{-k_{i} x\left(t+t^{\prime}\right)}\left(t t^{\prime}\right)^{\frac{n}{2}+k_{i}-\frac{1}{2}} \\
& K_{\frac{n}{2}-p-\frac{1}{2}}(t) K_{\frac{n}{2}-p-\frac{1}{2}}\left(t^{\prime}\right) d t d t^{\prime} d x<\infty
\end{aligned}
$$

or

$$
\begin{aligned}
& \sum_{i}\left(k_{i}+p\right)\left(k_{i}+n-p\right) c_{p, k_{i}}^{2}\left|c_{i}\right|^{2} \frac{k_{i}^{2 k_{i}-2 \epsilon} \Gamma^{2}\left(1+\frac{n}{2}+k_{i}\right)}{\Gamma^{2}\left(k_{i}+p+1\right) \Gamma^{2}\left(n+k_{i}-p\right)} \\
& \int_{k_{i}^{-1}}^{\infty} x^{2 k_{i}-1-2 \epsilon} \int_{0}^{\infty} \int_{0}^{\infty}\left(1-\frac{1}{k_{i}^{2} x^{2}}\right)^{k_{i}}\left(1+\frac{1}{k_{i} x}\right)^{-1-2 \epsilon} e^{-k_{i} x\left(t+t^{\prime}\right)}\left(t t^{\prime}\right)^{\frac{n}{2}+k_{i}-\frac{1}{2}} \\
& K_{\frac{n}{2}-p-\frac{1}{2}}(t) K_{\frac{n}{2}-p-\frac{1}{2}}\left(t^{\prime}\right) d t d t^{\prime} d x<\infty
\end{aligned}
$$


Formally taking $k_{i}$ large, we obtain

$$
\begin{aligned}
& \sum_{i}\left(k_{i}+p\right)\left(k_{i}+n-p\right) c_{p, k_{i}}^{2}\left|c_{i}\right|^{2} \frac{k_{i}^{2 k_{i}-2 \epsilon} \Gamma^{2}\left(1+\frac{n}{2}+k_{i}\right)}{\Gamma^{2}\left(k_{i}+p+1\right) \Gamma^{2}\left(n+k_{i}-p\right)} \\
& \int_{0}^{\infty} \int_{0}^{\infty} \int_{0}^{\infty} x^{2 k_{i}-1-2 \epsilon} e^{-k_{i} x\left(t+t^{\prime}\right)}\left(t t^{\prime}\right)^{\frac{n}{2}+k_{i}-\frac{1}{2}} \\
& K_{\frac{n}{2}-p-\frac{1}{2}}(t) K_{\frac{n}{2}-p-\frac{1}{2}}\left(t^{\prime}\right) d x d t d t^{\prime}<\infty,
\end{aligned}
$$

or

$$
\begin{aligned}
& \sum_{i}\left(k_{i}+p\right)\left(k_{i}+n-p\right) c_{p, k_{i}}^{2}\left|c_{i}\right|^{2} \frac{\Gamma\left(2 k_{i}-2 \epsilon\right) \Gamma^{2}\left(1+\frac{n}{2}+k_{i}\right)}{\Gamma^{2}\left(k_{i}+p+1\right) \Gamma^{2}\left(n+k_{i}-p\right)} \\
& \int_{0}^{\infty} \int_{0}^{\infty}\left(t+t^{\prime}\right)^{-2 k_{i}+2 \epsilon}\left(t t^{\prime}\right)^{\frac{n}{2}+k_{i}-\frac{1}{2}} K_{\frac{n}{2}-p-\frac{1}{2}}(t) K_{\frac{n}{2}-p-\frac{1}{2}}\left(t^{\prime}\right) d t d t^{\prime}<\infty .
\end{aligned}
$$

That is,

$$
\begin{aligned}
& \sum_{i}\left(k_{i}+p\right)\left(k_{i}+n-p\right) c_{p, k_{i}}^{2}\left|c_{i}\right|^{2} \frac{\Gamma\left(2 k_{i}-2 \epsilon\right) \Gamma^{2}\left(1+\frac{n}{2}+k_{i}\right)}{\Gamma^{2}\left(k_{i}+p+1\right) \Gamma^{2}\left(n+k_{i}-p\right)} 4^{-k_{i}} \\
& \int_{0}^{\infty} \int_{0}^{\infty}\left(\frac{t+t^{\prime}}{2 \sqrt{t t^{\prime}}}\right)^{-2 k_{i}}\left(t t^{\prime}\right)^{\frac{n}{2}-\frac{1}{2}}\left(t+t^{\prime}\right)^{2 \epsilon} K_{\frac{n}{2}-p-\frac{1}{2}}(t) K_{\frac{n}{2}-p-\frac{1}{2}}\left(t^{\prime}\right) d t d t^{\prime}<\infty .
\end{aligned}
$$

Making the change of variables $t=e^{u} v$ and $t^{\prime}=e^{-u} v$, we have

$$
\begin{aligned}
& \sum_{i}\left(k_{i}+p\right)\left(k_{i}+n-p\right) c_{p, k_{i}}^{2}\left|c_{i}\right|^{2} \frac{\Gamma\left(2 k_{i}-2 \epsilon\right) \Gamma^{2}\left(1+\frac{n}{2}+k_{i}\right)}{\Gamma^{2}\left(k_{i}+p+1\right) \Gamma^{2}\left(n+k_{i}-p\right)} 4^{-k_{i}} \\
& \int_{-\infty}^{\infty}(\cosh u)^{-2 k_{i}}(\cosh u)^{2 \epsilon} \int_{0}^{\infty} v^{n+2 \epsilon} K_{\frac{n}{2}-p-\frac{1}{2}}\left(e^{u} v\right) K_{\frac{n}{2}-p-\frac{1}{2}}\left(e^{-u} v\right) d v d u<\infty .
\end{aligned}
$$

From [11, (8) p. 325],

$$
\begin{aligned}
\int_{0}^{\infty} & v^{n+2 \epsilon} K_{\frac{n}{2}-p-\frac{1}{2}}\left(e^{u} v\right) K_{\frac{n}{2}-p-\frac{1}{2}}\left(e^{-u} v\right) d v= \\
& 2^{n+2 \epsilon+1} e^{-(2 n-2 p+2 \epsilon) u} \frac{\Gamma(n-p+\epsilon) \Gamma^{2}\left(\frac{1+n+2 \epsilon}{2}\right) \Gamma(1+p+\epsilon)}{8 \Gamma(1+n+2 \epsilon)} \\
& F\left(n-p+\epsilon, \frac{1+n+2 \epsilon}{2} ; 1+n+2 \epsilon ; 1-e^{-4 u}\right) .
\end{aligned}
$$

Using the asymptotics of the hypergeometric function from $[1,15.3 .6]$, one finds that for large $u$,

$$
(\cosh u)^{2 \epsilon} \int_{0}^{\infty} v^{n+2 \epsilon} K_{\frac{n}{2}-p-\frac{1}{2}}\left(e^{u} v\right) K_{\frac{n}{2}-p-\frac{1}{2}}\left(e^{-u} v\right) d v=O\left(e^{-2|u|}\right) .
$$


Thus we can apply steepest descent methods to (2.33) to obtain

$$
\begin{aligned}
& \sum_{i}\left(k_{i}+p\right)\left(k_{i}+n-p\right) c_{p, k_{i}}^{2}\left|c_{i}\right|^{2} \frac{\Gamma\left(2 k_{i}-2 \epsilon\right) \Gamma^{2}\left(1+\frac{n}{2}+k_{i}\right)}{\Gamma^{2}\left(k_{i}+p+1\right) \Gamma^{2}\left(n+k_{i}-p\right)} 4^{-k_{i}} \\
& k_{i}^{-\frac{1}{2}} \int_{0}^{\infty} v^{n+2 \epsilon} K_{\frac{n}{2}-p-\frac{1}{2}}^{2}(v) d v<\infty .
\end{aligned}
$$

Using the asymptotics of the gamma function [1, 6.1.39], we find

$$
\sum_{i} k_{i}^{-2 p-2 \epsilon}\left|c_{i}\right|^{2}<\infty
$$

Recalling (2.5), this is equivalent to saying that $\omega$ is Sobolev $\mathrm{H}^{-p-\epsilon}$-regular.

To justify passing from (2.29) to (2.30), it is enough to note that if $x \geq k_{i}^{-1}$ then

$$
\left(1-\frac{1}{k_{i}^{2} x^{2}}\right)^{k_{i}}\left(1+\frac{1}{k_{i} x}\right)^{-1-2 \epsilon}<1 .
$$

Thus we have uniform bounds in the preceding arguments.

Corollary 2. Suppose that $H^{n} / \Gamma$ has positive injectivity radius. Suppose that $\alpha$ is an $L^{2}$-harmonic $p$-form on $H^{n} / \Gamma, p \in\left[1, \frac{n}{2}\right]$. Then $\Phi_{p}^{-1}\left(\pi^{*} \alpha\right)$ is Sobolev $\mathrm{H}^{-p-\epsilon}$-regular for all $\epsilon>0$.

Proof. By elliptic theory [4, Prop. 1.3], there is a constant $r>0$ such that for all $m \in H^{n} / \Gamma,|\alpha(m)|$ is bounded in terms of the $L^{2}$-norm of $\alpha$ on the ball $B_{r}(m) \subset H^{n} / \Gamma$. Then $\pi^{*} \alpha$ is uniformly bounded on $H^{n}$. The corollary follows from Theorem 7 .

Theorem 2.A. If $\Gamma$ is cocompact then for any $p \in\left[1, \frac{n}{2}\right]$, there are isomorphisms between the following vector spaces :

$V_{1}=\left\{\right.$ Harmonic $p$-forms on $\left.H^{n} / \Gamma\right\}$.

$V_{2}=\left\{\Gamma\right.$-invariant exact $p$-hyperforms on $\left.S^{n-1}\right\}$.

$V_{3}=\left\{\Gamma\right.$-invariant exact $p$-currents on $S^{n-1}$ which are Sobolev $\mathrm{H}^{-p-\epsilon}$-regular for all $\epsilon>0\}$.

$V_{4}=\mathrm{H}^{p}\left(H^{n} / \Gamma, \mathbb{R}\right)$, the p-dimensional real cohomology group of $H^{n} / \Gamma$.

Proof. By standard Hodge theory, $V_{1} \cong V_{4}$. In particular, $V_{1}$ is finite-dimensional. By Corollary 2, there is an injection $V_{1} \rightarrow V_{3}$. There is an evident injection $V_{3} \rightarrow V_{2}$. By Gaillard's theorem [6, Théorème 2], if $\omega \in V_{2}$ then $\Phi_{p}(\omega)$ is a $\Gamma$ invariant closed and coclosed $p$-form on $H^{n}$. Hence $\Phi_{p}(\omega)=\pi^{*} \alpha$ for some closed and coclosed $p$-form $\alpha$ on $H^{n} / \Gamma$. Hence there is an injection $V_{2} \rightarrow V_{1}$. The theorem follows. 
Corollary 3. Suppose that there is a positive lower bound to the lengths of the nontrivial closed geodesics on $H^{n} / \Gamma$. Suppose that all of the cusps of $H^{n} / \Gamma$ have rank $n-1$. If $\alpha$ is an $L^{2}$-harmonic $p$-form on $H^{n} / \Gamma, p \in\left\{\frac{n-1}{2}, \frac{n}{2}\right\}$, then for all $\epsilon>0$, the hyperform $\Phi_{p}^{-1}\left(\pi^{*} \alpha\right)$ is Sobolev $\mathrm{H}^{-p-\epsilon}$-regular.

Proof. For some $\mu>0$ less than the Margulis constant of $H^{n}$, the $\mu$-thin part of $H^{n} / \Gamma$ has a finite number of compact components. By the proof of Corollary $2, \alpha$ is bounded on the $\mu$-thick part of $H^{n} / \Gamma$. It follows from [12, Theorem 4.12] that $\alpha$ is bounded on the cusps of $H^{n} / \Gamma$. The corollary follows from Theorem 7 .

Theorem 3. In the case $n=3$, suppose that there is a positive lower bound to the lengths of the nontrivial closed geodesics on $H^{3} / \Gamma$. Let $\alpha$ be an $L^{2}$-harmonic 1 -form on $H^{3} / \Gamma$. Then for all $\epsilon>0$, the hyperform $\Phi_{1}^{-1}\left(\pi^{*} \alpha\right)$ is Sobolev $\mathrm{H}^{-1-\epsilon}$ regular.

Proof. Following the line of proof of Corollary 3, it suffices to analyze the asymptotics of an $L^{2}$-harmonic 1-form $\omega$ on a rank-1 cusp. We can take a neighborhood of such a cusp to be the quotient of

$$
\left\{(x, y, z): y^{2}+z^{2} \geq R, z \geq 0\right\}
$$

by the group generated by $x \rightarrow x+2 \pi$, for some $R>0$. We follow the analysis of [12, Section 4], with care for constants. Make a change of coordinates to $y=r \cos \theta$, $z=r \sin \theta$, with $r \in[R, \infty), \theta \in\left(-\frac{\pi}{2}, \frac{\pi}{2}\right)$. The Riemannian metric in these coordinates is

$$
d s^{2}=\frac{d x^{2}}{r^{2} \cos ^{2} \theta}+\frac{d r^{2}}{r^{2} \cos ^{2} \theta}+\frac{d \theta^{2}}{\cos ^{2} \theta},
$$

with volume form $d \mathrm{vol}=\frac{d x d r d \theta}{r^{2} \cos ^{3} \theta}$.

Let

$$
\omega=\alpha_{0} d \theta+\alpha_{1} d x+\beta_{0} d r
$$

be an $L^{2}$-harmonic 1 -form on the cusp. Then

$$
\int\left(r^{-2}\left|\alpha_{0}\right|^{2}+\left|\alpha_{1}\right|^{2}+\left|\beta_{0}\right|^{2}\right) \frac{d x d r d \theta}{\cos \theta}<\infty
$$

The equations $d \omega=d^{*} \omega=0$ become

$$
\begin{aligned}
0 & =\partial_{x} \alpha_{0}-\partial_{\theta} \alpha_{1}=\partial_{r} \alpha_{0}-\partial_{\theta} \beta_{0}=\partial_{r} \alpha_{1}-\partial_{x} \beta_{0} \\
& =\cos \theta \partial_{\theta}\left(\frac{\alpha_{0}}{\cos \theta}\right)+r^{2} \partial_{x} \alpha_{1}+r^{2} \partial_{r} \beta_{0} .
\end{aligned}
$$


From these equations, one obtains the Laplacian-type equations

$$
\begin{aligned}
& -\partial_{r}^{2} \alpha_{0}-\partial_{x}^{2} \alpha_{0}-\frac{1}{r^{2}} \partial_{\theta}\left(\cos \theta \partial_{\theta}\left(\frac{\alpha_{0}}{\cos \theta}\right)\right)=0 \\
& -\partial_{r}^{2} \alpha_{1}-\partial_{x}^{2} \alpha_{1}-\frac{1}{r^{2}} \cos \theta \partial_{\theta}\left(\frac{1}{\cos \theta} \partial_{\theta} \alpha_{1}\right)=0 \\
& -\partial_{r}^{2} \beta_{0}-\partial_{x}^{2} \beta_{0}-\frac{1}{r^{2}} \cos \theta \partial_{\theta}\left(\frac{1}{\cos \theta} \partial_{\theta} \beta_{0}\right)=-\frac{2}{r^{3}} \cos \theta \partial_{\theta}\left(\frac{\alpha_{0}}{\cos \theta}\right) .
\end{aligned}
$$

We first analyze the second equation in (2.44). Given a function $f \in C^{\infty}\left(-\frac{\pi}{2}, \frac{\pi}{2}\right)$, put

$$
L f=-\cos \theta \partial_{\theta}\left(\frac{1}{\cos \theta} \partial_{\theta} f\right)
$$

Then $L$ is the self-adjoint operator coming from the Dirichlet form on $L^{2}\left(\left(-\frac{\pi}{2}, \frac{\pi}{2}\right)\right.$, $\left.\frac{1}{\cos \theta} d \theta\right)$. Making the change of variable $u=\sin \theta$, the eigenfunction equation $L f=\lambda f$ becomes

$$
-\left(1-u^{2}\right) f^{\prime \prime}(u)=\lambda f
$$

The square-integrable solutions to this have $\lambda=(q+1)(q+2)$ with $q \in \mathbb{Z} \cap[0, \infty)$. The corresponding eigenfunction is given in terms of ultraspherical polynomials $[1,22.6 .6]$ by

$$
f_{q}(u)=\left(1-u^{2}\right) C_{q}^{3 / 2}(u) .
$$

Explicitly, $f_{q}(u)$ is proportionate to $\frac{d^{q}}{d u^{q}}\left(\left(1-u^{2}\right)^{q+1}\right)$.

Performing separation of variables on the second equation in (2.44), suppose that

$$
\alpha_{1}(x, r, \theta)=e^{i m x} g(r) f_{q}(\theta)
$$

with $m \in \mathbb{Z}$. Then

$$
-g^{\prime \prime}+m^{2} g+\frac{(q+1)(q+2)}{r^{2}} g=0 .
$$

If $m \neq 0$ then $g$ decreases exponentially fast in $r$. Suppose that $m=0$. One finds that for large $r, g(r) \sim r^{q+2}$ or $g(r) \sim r^{-q-1}$. For $\omega$ to be square-integrable, one must have $g(r) \sim r^{-q-1}$. If $q>0$ then $\left|\alpha_{1} d x\right|=r \cos \theta|g(r)|\left|f_{q}(\theta)\right|$ decays polynomially fast in $r$. In the critical case $q=0,\left|\alpha_{1} d x\right|$ remains bounded in $r$.

Next, put

$$
L^{\prime} f=-\partial_{\theta}\left(\cos \theta \partial_{\theta}\left(\frac{f}{\cos \theta}\right)\right) .
$$

and $\widehat{L}=\frac{1}{\cos \theta} \circ L^{\prime} \circ \cos \theta$. Then $\widehat{L}$ is the self-adjoint operator coming from the Dirichlet form on $L^{2}\left(\left(-\frac{\pi}{2}, \frac{\pi}{2}\right), \cos \theta d \theta\right)$. It has a nonnegative discrete spectrum starting at 0 , and hence so does $L^{\prime}$. Suppose that $f(\theta)$ is an eigenfunction of $L^{\prime}$ 
with eigenvalue $\lambda \geq 0$. Performing separation of variables on the first equation in (2.44), suppose that

$$
\alpha_{0}(x, r, \theta)=e^{i m x} g(r) f(\theta)
$$

with $m \in \mathbb{Z}$. Then

$$
-g^{\prime \prime}+m^{2} g+\frac{\lambda}{r^{2}} g=0
$$

If $m \neq 0$ then $g$ decreases exponentially fast in $r$. Suppose that $m=0$. One finds that for large $r, g(r) \sim r^{\frac{1 \pm \sqrt{1+4 \lambda}}{2}}$. For $\omega$ to be square-integrable, one must have $g(r) \sim r^{\frac{1-\sqrt{1+4 \lambda}}{2}}$. If $\lambda>0$ then $\left|\alpha_{0} d \theta\right|=\cos \theta|g(r)||f(\theta)|$ decays like a power in $r$. In the critical case $\lambda=0,\left|\alpha_{0} d \theta\right|$ remains bounded in $r$.

Finally, one can analyze the third equation in (2.44), an inhomogeneous equation, by similar methods. The upshot is that $|\omega|$ is bounded on the rank-1 cusp.

Proposition 1. Suppose that there is a positive lower bound to the lengths of the nontrivial closed geodesics on $H^{n} / \Gamma$. Let $\alpha$ be an $L^{2}$-harmonic p-form on $H^{n} / \Gamma$, $p \in\left[1, \frac{n}{2}\right]$. Then $\Phi_{p}^{-1}\left(\pi^{*} \alpha\right)$ is a current.

Proof. For some $\mu>0$ less than the Margulis constant of $H^{n}$, the $\mu$-thin part of $H^{n} / \Gamma$ has a finite number of compact components. As in the proof of Corollary 2 , there is a uniform upper bound for $|\alpha|$ on the $\mu$-thick part of $H^{n} / \Gamma$. On each cuspidal component of the $\mu$-thin part, $|\alpha|$ has at most exponential growth, with a uniform exponential constant $[12$, Section 4]. The result follows from $[6$, Théorème $3]$.

Proposition 2. For $r \in(0,1)$, let $i_{r}: S^{n-1} \rightarrow S^{n-1}(r)$ be the embedding of $S^{n-1}$ as the r-sphere around 0 in the ball model of $H^{n}$. As in [6, p. 586], put

$$
C_{p}=\frac{2^{p}}{n} \frac{\Gamma(n-2 p+1) \Gamma\left(\frac{n}{2}+1\right)}{\Gamma(n-p) \Gamma\left(\frac{n}{2}-p+1\right)} .
$$

Let $\omega$ be an exact $p$-current on $S^{n-1}$. Then as $r \rightarrow 1$, the forms $i_{r}^{*} \Phi_{p}(\omega)$ converge to $C_{p} \omega$ in the sense of convergence of currents.

Proof. From (2.6),

$$
i_{r}^{*} \Phi_{p}(\omega)=\sum_{i=1}^{\infty} c_{i} \frac{\left(k_{i}+p\right)\left(k_{i}+n-p\right)}{2} c_{p, k_{i}} r^{p-1+k_{i}} \frac{r}{k_{i}+p} F_{p-1, k_{i}}\left(r^{2}\right) d \alpha_{i} .
$$

Given a smooth form $\eta \in \Omega^{p}\left(S^{n-1}\right)$, let $\Pi(\eta)$ be the projection of $\eta$ onto the square-integrable exact $p$-forms on $S^{n-1}$. Then $\Pi(\eta)$ is also smooth and has a Fourier expansion

$$
\Pi(\eta)=\sum_{i=1}^{\infty} a_{i} d \alpha_{i}
$$


with $\sum_{i=1}^{\infty} k_{i}^{N}\left|a_{i}\right|^{2}<\infty$ for all $N \in \mathbb{Z}^{+}$. The pairing

$$
\left\langle i_{r}^{*} \Phi_{p}(\omega), \eta\right\rangle=\int_{S^{n-1}} i_{r}^{*} \Phi_{p}(\omega) \wedge \overline{* \eta}
$$

is given by

$$
\left\langle i_{r}^{*} \Phi_{p}(\omega), \eta\right\rangle=\sum_{i=1}^{\infty} \overline{a_{i}} c_{i} \frac{\left(k_{i}+p\right)\left(k_{i}+n-p\right)}{2} c_{p, k_{i}} r^{p-1+k_{i}} \frac{r}{k_{i}+p} F_{p-1, k_{i}}\left(r^{2}\right)
$$

Then

$$
\begin{aligned}
\left\langle i_{1}^{*} \Phi_{p}(\omega), \eta\right\rangle= & \sum_{i=1}^{\infty} \overline{a_{i}} c_{i} \frac{\left(k_{i}+p\right)\left(k_{i}+n-p\right)}{2} c_{p, k_{i}} \frac{1}{k_{i}+p} \\
& \frac{\Gamma\left(1+\frac{n}{2}+k_{i}\right) \Gamma(1-2 p+n)}{\Gamma\left(1-p+n+k_{i}\right) \Gamma\left(1-p+\frac{n}{2}\right)} \\
= & C_{p} \sum_{i=1}^{\infty} \overline{a_{i}} c_{i} \\
= & C_{p}\langle\omega, \eta\rangle .
\end{aligned}
$$

As $\omega$ is a current, $\sum_{i=1}^{\infty} k_{i}^{N}\left|a_{i}\right|\left|c_{i}\right|<\infty$ for all $N \in \mathbb{Z}^{+}$.

Lemma 2. As $r$ increases from 0 to 1 , the expression $r^{p-1+k_{i}} \frac{r}{k_{i}+p} F_{p-1, k_{i}}\left(r^{2}\right)$ increases monotonically from 0 to $\frac{1}{k_{i}+p} \frac{\Gamma\left(1+\frac{n}{2}+k_{i}\right) \Gamma(1-2 p+n)}{\Gamma\left(1-p+n+k_{i}\right) \Gamma\left(1-p+\frac{n}{2}\right)}$.

Proof. The fact that the right-hand-side of (2.6) is closed implies that

$$
\frac{d}{d r}\left(r^{p-1+k_{i}} \frac{r}{k_{i}+p} F_{p-1, k_{i}}\left(r^{2}\right)\right)=r^{p-1+k_{i}}\left(1-r^{2}\right) F_{p, k_{i}}\left(r^{2}\right) .
$$

(Of course, this can be checked directly.) From [1, 15.3.3],

$$
\begin{aligned}
F_{p, k_{i}}\left(r^{2}\right) & =F\left(1+p-\frac{n}{2}, 1+p+k_{i} ; 1+\frac{n}{2}+k_{i} ; r^{2}\right) \\
& =\left(1-r^{2}\right)^{n-1-2 p} F\left(n+k_{i}-p, \frac{n}{2}-p ; 1+\frac{n}{2}+k_{i} ; r^{2}\right) .
\end{aligned}
$$

As the arguments of $F\left(n+k_{i}-p, \frac{n}{2}-p ; 1+\frac{n}{2}+k_{i} ; r^{2}\right)$ are all nonnegative, the lemma follows.

Proposition 2 now follows from dominated convergence. 
Proposition 3. Suppose that $\alpha$ is an $L^{2}$-harmonic $p$-form on $H^{n} / \Gamma, p \in\left[1, \frac{n}{2}\right)$. Suppose that $\Phi_{p}^{-1}\left(\pi^{*} \alpha\right)$ is a current. Then $\Phi_{p}^{-1}\left(\pi^{*} \alpha\right)$ is supported on the limit set $\Lambda$ of $\Gamma$.

Proof. Given a smooth form $\phi \in \Omega^{p}\left(S^{n-1}\right)$ with relatively compact support in $S^{n-1}-\Lambda$, Proposition 2 implies that

$$
\lim _{r \rightarrow 1}\left\langle i_{r}^{*} \pi^{*} \alpha, \phi\right\rangle=C_{p}\left\langle\Phi_{p}^{-1}\left(\pi^{*} \alpha\right), \phi\right\rangle .
$$

If $\Lambda=\emptyset$, we assume that $\operatorname{supp}(\phi) \neq S^{n-1}$; this is sufficient for the argument. Then we can use an upper-half-space model for $H^{n}$, with $\operatorname{supp}(\phi) \subset \mathbb{R}^{n-1}$. Put $V=\operatorname{supp}(\phi) \times(0, \infty) \subset H^{n}$. Using the coordinates $\left(x_{1}, \ldots, x_{n-1}, y\right)$ for $H^{n}$, let us write $\widetilde{\alpha}=a(x, y)+d y \wedge b(x, y)$. Then [12, Theorem 4.3] states that on $V$, as $y \rightarrow 0$,

and

$$
a= \begin{cases}a_{00}(x) y^{n-2 p-1}+O\left(y^{n-2 p} \log (y)\right) & \text { if } p<\frac{n-1}{2} \\ a_{01}(x) y^{2} \log (y)+O\left(y^{2}\right) & \text { if } p=\frac{n-1}{2}\end{cases}
$$

$$
b= \begin{cases}b_{01}(x) y^{n-2 p} \log (y)+O\left(y^{n-2 p}\right) & \text { if } p<\frac{n-1}{2}, \\ b_{00}(x) y+O\left(y^{2} \log (y)\right) & \text { if } p=\frac{n-1}{2} .\end{cases}
$$

(The statement of [12, Theorem 4.3] should read " $y \rightarrow 0$ ".) As $r \rightarrow 1$, the intersections $S^{n-1}(r) \cap V$ asymptotically approach the horosphere pieces

$$
\left\{\left(x_{1}, \ldots, x_{n-1}, y\right) \in H^{n}: y=\frac{1-r}{1+r}\right\} \cap V .
$$

It follows that $\left\langle\Phi_{p}^{-1}\left(\pi^{*} \alpha\right), \phi\right\rangle=0$ for all such $\phi$, from which the proposition follows.

Remark. The analog of Proposition 3 is false if $p=\frac{n}{2}$. This can be seen in the case $\Gamma=\{e\}$ using Theorem 1 .

We give a partial converse to Proposition 3, in the case of convex-cocompact groups.

Theorem 2.B. If $\Gamma$ is convex-cocompact then for any $p \in\left[1, \frac{n-1}{2}\right)$, there are isomorphisms between the following vector spaces :

$V_{1}=\left\{L^{2}\right.$-harmonic $p$-forms on $\left.H^{n} / \Gamma\right\}$.

$V_{2}=\left\{\Gamma\right.$-invariant exact $p$-hyperforms on $S^{n-1}$ which are supported on the limit set\}.

$V_{3}=\left\{\Gamma\right.$-invariant exact $p$-currents on $S^{n-1}$ which are supported on the limit set and which are Sobolev $\mathrm{H}^{-p-\epsilon}$-regular for all $\left.\epsilon>0\right\}$.

$V_{4}=\mathrm{H}_{c}^{p}\left(H^{n} / \Gamma, \mathbb{R}\right)$, the p-dimensional real compactly-supported cohomology group of $H^{n} / \Gamma$. 
Proof. By [12], $V_{1} \cong V_{4}$. In particular, $V_{1}$ is finite-dimensional. By Gaillard's theorem [6, Théorème 2], Corollary 2 and Proposition 3, there are injections $V_{1} \rightarrow$ $V_{3} \rightarrow V_{2}$. It remains to show that there is an injection $V_{2} \rightarrow V_{1}$. In view of Gaillard's theorem, it suffices to show that if $\omega \in V_{2}$ then $\Phi_{p}(\omega)$ descends to a form which is square-integrable on $H^{n} / \Gamma$. If $\Gamma$ is cocompact then this is automatic, so assume that $\Gamma$ is not cocompact. As $\Omega / \Gamma$ is compact, we can find a fundamental domain $F$ for the action of $\Gamma$ on $H^{n}$ such that $\bar{F} \cap S^{n-1}$ is disjoint from $\Lambda$. Take an upper-half-space model for $H^{n}$ with $\infty \in \Omega$. In terms of the upper-half-space coordinates $\left(x_{1}, \ldots, x_{n-1}, y\right)$, [6, Lemme 3$]$ implies that near $y=0$,

$$
\left.\Phi_{p}(\omega)\right|_{F}=y^{n-2 p-1} \phi(x, y)
$$

where the $p$-form $\phi(x, y)$ is continuous up to $y=0$. It follows that $\int_{F}\left|\Phi_{p}(\omega)\right|^{2} d$ vol $<\infty$.

\section{1-Forms}

In this section we look in more detail at the case of $L^{2}$-harmonic 1-forms on convexcocompact hyperbolic manifolds. If the hyperbolic manifold is compact, we show that the Sobolev regularity estimate of Theorem 2.A is sharp. If the hyperbolic manifold is convex-cocompact but not compact, we show how to construct its $L^{2}$-harmonic 1-forms explicitly in terms of the harmonic extension of functions. In this case, we show that the Sobolev regularity estimate of Corollary 2 can be slightly improved.

Proposition 4. Suppose that $\Gamma$ is cocompact. For $\epsilon>0$, let $V_{\epsilon}^{\Gamma}$ be the $\Gamma$-invariant subspace of the function space $\mathrm{H}^{-\epsilon}\left(S^{n-1}\right) / \mathbb{C}$. Then $V_{\epsilon}^{\Gamma}$ is isomorphic to $\mathrm{H}^{1}(\Gamma ; \mathbb{C})$.

Proof. We first define linear maps $I: \mathrm{H}^{1}(\Gamma ; \mathbb{C}) \rightarrow V_{\epsilon}^{\Gamma}$ and $J: V_{\epsilon}^{\Gamma} \rightarrow \mathrm{H}^{1}(\Gamma ; \mathbb{C})$. To define $I$, given $x \in \mathrm{H}^{1}(\Gamma ; \mathbb{C})=\mathrm{H}^{1}\left(H^{n} / \Gamma ; \mathbb{C}\right)$, let $\alpha \in \Omega^{1}\left(H^{n} / \Gamma\right)$ be the harmonic 1form which represents $x$. Put $\widetilde{\alpha}=\pi^{*} \alpha$. By Theorem $7, \Phi_{1}^{-1}(\widetilde{\alpha})$ is an exact $\mathrm{H}^{-1-\epsilon}$ regular $\Gamma$-invariant 1 -form on $S^{n-1}$. Choose $f \in \mathrm{H}^{-\epsilon}\left(S^{n-1}\right)$ so that $\Phi_{1}^{-1}(\widetilde{\alpha})=d f$. Then for all $\gamma \in \Gamma$,

$$
d(f-\gamma \cdot f)=d f-\gamma \cdot d f=0 .
$$

Thus

$$
f-\gamma \cdot f=c(\gamma)
$$

for some $c(\gamma) \in \mathbb{C}$. Put $I(x)=f \bmod \mathbb{C}$.

To define $J$, given $\bar{f} \in V_{\epsilon}^{\Gamma}$, let $f \in \mathrm{H}^{-\epsilon}\left(S^{n-1}\right)$ be a representative of $\bar{f}$, not necessarily $\Gamma$-invariant. As $\frac{\epsilon}{f}$ is $\Gamma$-invariant, for each $\gamma \in \Gamma$ there is a $c(\gamma) \in \mathbb{C}$ such that $f-\gamma \cdot f=c(\gamma)$. As

$c\left(\gamma_{1} \gamma_{2}\right)=f-\left(\gamma_{1} \gamma_{2}\right) \cdot f=\left(f-\gamma_{1} \cdot f\right)+\gamma_{1} \cdot\left(f-\gamma_{2} \cdot f\right)=c\left(\gamma_{1}\right)+\gamma_{1} \cdot c\left(\gamma_{2}\right)=c\left(\gamma_{1}\right)+c\left(\gamma_{2}\right)$, 
we have a cocycle $c: \Gamma \rightarrow \mathbb{C}$. Put $J(\bar{f})=[c]$.

We show that $J \circ I$ is the identity. It suffices to show that the cocycle $c$ of (3.2) represents $x \in \mathrm{H}^{1}(\Gamma ; \mathbb{C})$. For this, it suffices to show that for all $\gamma \in \Gamma$,

$$
c(\gamma)=\int_{C_{\gamma}} \alpha,
$$

where $C_{\gamma}$ is a closed curve on $H^{n} / \Gamma$ in the homotopy class of $\gamma \in \pi_{1}\left(H^{n} / \Gamma\right)$ and $\alpha \in \Omega^{1}\left(H^{n} / \Gamma\right)$ is the harmonic representative of $x$. Let $\widetilde{C}_{\gamma}$ be a lift of $C_{\gamma}$ to $H^{n}$, ending at a point $m \in H^{n}$ and starting at $\gamma^{-1} \cdot m$. Then

$$
\begin{aligned}
\int_{C_{\gamma}} \alpha & =\int_{\widetilde{C}_{\gamma}} \widetilde{\alpha}=\int_{\widetilde{C}_{\gamma}} \Phi_{1}(d f)=\int_{\widetilde{C}_{\gamma}} d \Phi_{0}(f)=\left(\Phi_{0}(f)\right)(m)-\left(\Phi_{0}(f)\right)\left(\gamma^{-1} \cdot m\right) \\
& =\left(\Phi_{0}(f)-\gamma \cdot \Phi_{0}(f)\right)(m)=\left(\Phi_{0}(f-\gamma \cdot f)\right)(m)=\left(\Phi_{0}(c(\gamma))\right)(m)=c(\gamma) .
\end{aligned}
$$

This shows that $J \circ I$ is the identity. To see that $I \circ J$ is the identity, given $\bar{f} \in V_{\epsilon}^{\Gamma}$, let $f \in \mathrm{H}^{-\epsilon}\left(S^{n-1}\right)$ be a representative of $\bar{f}$, not necessarily $\Gamma$-invariant. Define $\widetilde{\alpha}=\Phi_{1}(d f)$. Then $\widetilde{\alpha}$ is a smooth $\Gamma$-invariant harmonic 1 -form on $H^{n}$ and projects to a harmonic 1-form $\alpha \in \Omega^{1}\left(H^{n} / \Gamma\right)$. By the same sort of calculation as in (3.5), one finds that $J(\bar{f})=[\alpha]$ in $\mathrm{H}^{1}(\Gamma ; \mathbb{C})$. By construction, $I([\alpha])=\bar{f}$. Thus $I \circ J$ is the identity.

Theorem 4.A. Suppose that $\Gamma$ is cocompact. Let $\alpha$ be a nonzero harmonic 1-form on $H^{n} / \Gamma$. Then $\Phi_{1}^{-1}\left(\pi^{*} \alpha\right)$ is not Sobolev $\mathrm{H}^{-1}$-regular.

Proof. Suppose that $\Phi_{1}^{-1}\left(\pi^{*} \alpha\right)$ is Sobolev $\mathrm{H}^{-1}$-regular. Then $\Phi_{1}^{-1}\left(\pi^{*} \alpha\right)=d f$ for some $f \in \mathrm{L}^{2}\left(S^{n-1}\right)$. Extending the proof of Proposition 4 to the case $\epsilon=0$, the equivalence class $\bar{f}$ of $f$ in $\mathrm{L}^{2}\left(S^{n-1}\right) / \mathbb{C}$ is $\Gamma$-invariant and satisfies $J(\bar{f})=[\alpha]$. As $\Gamma$ acts ergodically on $S^{n-1}$, we must have $\bar{f}=0$ and hence $[\alpha]$ vanishes in $\mathrm{H}^{1}\left(H^{n} / \Gamma ; \mathbb{C}\right)$, which is a contradiction.

We now consider groups $\Gamma$ which are convex-cocompact but not compact. First, we prove some generalities about the relationship between compactly-supported cohomology and $L^{2}$-cohomology.

Let $M$ be a complete connected oriented Riemannian manifold. Let $\mathrm{H}_{(2)}^{p}(M)$ be the $p$-th (reduced) $L^{2}$-cohomology group of $M$. It is isomorphic to $\operatorname{Ker}\left(\triangle_{p}\right)$. There is a map $i: \mathrm{H}_{c}^{p}(M ; \mathbb{C}) \rightarrow \mathrm{H}_{(2)}^{p}(M)$. In general, $i$ is not injective; think of $M=\mathbb{R}^{n}$. However, it is true, and well-known, that $i$ always induces an injection of $\operatorname{Im}\left(\mathrm{H}_{c}^{p}(M ; \mathbb{C}) \rightarrow \mathrm{H}^{p}(M ; \mathbb{C})\right)$ into $\mathrm{H}_{(2)}^{p}(M)[9$, Prop. 4]. The next result gives a sufficient condition for $i$ to be injective on all of $\mathrm{H}_{c}^{1}(M ; \mathbb{C})$. Recall that there is a notion of the space of ends of $M$, and of an end being contained in an open set $U \subset M$; see, for example, $[3, \S 1.2]$. 
Proposition 5. Suppose that for every end e of $M$, every open set $U$ containing e has infinite volume. Suppose that $M$ has a Green's operator $G: C_{0}^{\infty}(M) \rightarrow L^{2}(M)$ such that $\triangle \circ G=\mathrm{Id}$. Then $i: \mathrm{H}_{c}^{1}(M ; \mathbb{C}) \rightarrow \mathrm{H}_{(2)}^{1}(M)$ is injective.

Proof. We have the decomposition

$$
\mathrm{H}_{c}^{1}(M ; \mathbb{C})=\left(\operatorname{Ker}\left(\mathrm{H}_{c}^{1}(M ; \mathbb{C}) \rightarrow \mathrm{H}^{1}(M ; \mathbb{C})\right)\right) \oplus\left(\operatorname{Im}\left(\mathrm{H}_{c}^{1}(M ; \mathbb{C}) \rightarrow \mathrm{H}^{1}(M ; \mathbb{C})\right)\right) .
$$

We first show that $i$ is injective on $\operatorname{Ker}\left(\mathrm{H}_{c}^{1}(M ; \mathbb{C}) \rightarrow \mathrm{H}^{1}(M ; \mathbb{C})\right)$. A representative of $\operatorname{Ker}\left(\mathrm{H}_{c}^{1}(M ; \mathbb{C}) \rightarrow \mathrm{H}^{1}(M ; \mathbb{C})\right)$ is a closed compactly-supported 1-form $\alpha$ such that $\alpha=d f$ for some function $f$. By construction, $f$ is locally constant outside of a compact subset of $M$ and so gives a function on the space of ends of $M$. Now $d(f-G \triangle f)$ is a harmonic 1 -form on $M$. As

$$
\langle d G \triangle f, d G \triangle f\rangle=\langle G \triangle f, \triangle f\rangle,
$$

we have that $d(f-G \triangle f)$ is square-integrable. The map $\alpha \rightarrow d(f-G \triangle f)$ describes $i$ on $\operatorname{Ker}\left(\mathrm{H}_{c}^{1}(M ; \mathbb{C}) \rightarrow \mathrm{H}^{1}(M ; \mathbb{C})\right)$. To see that it is injective, suppose that $d(f-$ $G \triangle f)=0$. Then $f-G \triangle f$ is constant. As $G \triangle f \in L^{2}(M)$, the volume assumption implies that $f$, as a function on the space of ends of $M$, is a constant $c$. Then $f-c$ is compactly-supported on $M$, with $d(f-c)=\alpha$, so $[\alpha]=0$ in $\mathrm{H}_{c}^{1}(M ; \mathbb{C})$. In summary, we have realized an injection of $\operatorname{Ker}\left(\mathrm{H}_{c}^{1}(M ; \mathbb{C}) \rightarrow \mathrm{H}^{1}(M ; \mathbb{C})\right)$ into $\mathrm{H}_{(2)}^{1}(M)$.

It remains to show that

$$
i\left(\operatorname{Ker}\left(\mathrm{H}_{c}^{1}(M ; \mathbb{C}) \rightarrow \mathrm{H}^{1}(M ; \mathbb{C})\right)\right) \cap i\left(\operatorname{Im}\left(\mathrm{H}_{c}^{1}(M ; \mathbb{C}) \rightarrow \mathrm{H}^{1}(M ; \mathbb{C})\right)\right)=0 .
$$

Suppose that $d(f-G \triangle f)$ is nonzero and lies in the image, under $i$, of $\operatorname{Im}\left(\mathrm{H}_{c}^{1}(M ; \mathbb{C})\right.$ $\left.\rightarrow \mathrm{H}^{1}(M ; \mathbb{C})\right)$. Then $d(f-G \triangle f)=\omega \bmod \overline{\operatorname{Im}(d)}$ for some closed compactlysupported 1-form $\omega$. Furthermore, by assumption, there is a closed compactlysupported $(\operatorname{dim}(M)-1)$-form $\eta$ such that $\int_{M} \omega \wedge \eta=1$. However, $\int_{M} d(f-$ $G \triangle f) \wedge \eta=0$. It follows that

$$
i\left(\operatorname{Ker}\left(\mathrm{H}_{c}^{1}(M ; \mathbb{C}) \rightarrow \mathrm{H}^{1}(M ; \mathbb{C})\right)\right) \cap i\left(\operatorname{Im}\left(\mathrm{H}_{c}^{1}(M ; \mathbb{C}) \rightarrow \mathrm{H}^{1}(M ; \mathbb{C})\right)\right)=0 .
$$

This proves the proposition.

Suppose that $\Gamma$ is convex-cocompact but not cocompact. Then $H^{n} / \Gamma$ satisfies the hypotheses of Proposition 5 and so $i: \mathrm{H}_{c}^{1}\left(H^{n} / \Gamma ; \mathbb{C}\right) \rightarrow \mathrm{H}_{(2)}^{1}\left(H^{n} / \Gamma\right)$ is injective. For the rest of this section, we assume that $n>2$. It follows from [12, Theorem $3.13]$ that $i$ is an isomorphism. This essentially comes from the fact that given an $L^{2}$-harmonic 1-form $\omega$ on $H^{n} / \Gamma$, one can apply the Poincaré Lemma from infinity 
to homotop $\omega$ to something with compact support. We show how to construct the $L^{2}$-harmonic 1-forms on $H^{n} / \Gamma$ explicitly.

Lemma 3. There is an isomorphism between $\mathrm{H}_{c}^{1}\left(H^{n} / \Gamma ; \mathbb{C}\right)$ and the quotient space

$$
\begin{gathered}
W=\left\{(f, c) \in C^{\infty}(\Omega) \times \mathrm{H}^{1}(\Gamma ; \mathbb{C}): f \text { is locally-constant and for all } \gamma \in \Gamma,\right. \\
f-\gamma \cdot f=c(\gamma)\} / \mathbb{C} .
\end{gathered}
$$

(Here $\mathbb{C}$ acts by addition on $C^{\infty}(\Omega)$ and fixes $\mathrm{H}^{1}(\Gamma ; \mathbb{C})$.)

Proof. Given $x \in \mathrm{H}_{c}^{1}\left(H^{n} / \Gamma ; \mathbb{C}\right)$, represent it by a smooth closed compact-supported 1 -form $\alpha \in \Omega^{1}\left(H^{n} / \Gamma\right)$. Put $\widetilde{\alpha}=\pi^{*} \alpha$. As $\alpha$ is compactly-supported, we can extend $\widetilde{\alpha}$ continuously by zero to become a closed 1 -form on $H^{n} \cup \Omega$. Fix a point $s \in \Omega$. Define $f: \Omega \rightarrow \mathbb{C}$ by

$$
f(z)=\int_{\widetilde{C}} \widetilde{\alpha}
$$

where $\widetilde{C}$ is a curve in $H^{n} \cup \Omega$ from $s$ to $z$. Then

$$
(f-\gamma \cdot f)(z)=\int_{\widetilde{C}^{\prime}} \widetilde{\alpha}
$$

where $\widetilde{C}^{\prime}$ is a curve in $H^{n} \cup \Omega$ from $\gamma^{-1} \cdot z$ to $z$. Now $\widetilde{C}^{\prime}$ projects to a closed curve $C^{\prime}$ on the compact manifold-with-boundary $\left(H^{n} \cup \Omega\right) / \Gamma$. Then

$$
(f-\gamma \cdot f)(z)=\int_{C^{\prime}} \alpha .
$$

It follows that $f-\gamma \cdot f=c(\gamma)$, where $c$ is the image of $x$ in $^{1}\left(\left(H^{n} \cup \Omega\right) / \Gamma ; \mathbb{C}\right) \cong$ $\mathrm{H}^{1}(\Gamma ; \mathbb{C})$. A different choice of $s$ changes $f$ by a constant.

Conversely, given $(f, c) \in W$, fix a point $m_{0} \in H^{n} / \Gamma$. Let $R$ be large enough that the convex core of $H^{n} / \Gamma$ lies within $B_{R}\left(m_{0}\right)$. Let $\phi:[0, \infty) \rightarrow \mathbb{R}$ be a smooth function which is monotonically nonincreasing, identically one on $[0, R]$ and identically zero on $[R+1, \infty)$. Let $\eta \in C^{\infty}\left(H^{n}\right)$ be the lift to $H^{n}$ of $\phi\left(d\left(m_{0}, \cdot\right)\right) \in$ $C^{\infty}\left(H^{n} / \Gamma\right)$. Extend $f$ inward to a locally-constant smooth function $F:\left(H^{n}-\right.$ $\left.\pi^{-1}\left(B_{R}\left(m_{0}\right)\right)\right) \rightarrow \mathbb{C}$. Put $\widetilde{\alpha}=d((1-\eta) F)$ on $H^{n}-\pi^{-1}\left(B_{R}\left(m_{0}\right)\right)$ and extend it by zero to $H^{n}$. Then $\widetilde{\alpha}$ is a closed $\Gamma$-invariant 1 -form on $H^{n}$ which descends to a closed 1-form $\alpha \in \Omega^{1}\left(H^{n} / \Gamma\right)$ with support in $B_{R+1}\left(m_{0}\right)$, and hence an element $[\alpha] \in \mathrm{H}_{c}^{1}\left(H^{n} / \Gamma ; \mathbb{C}\right)$.

One can check that these two maps are inverses. We omit the details.

The map $W \rightarrow \mathrm{H}^{1}\left(H^{n} / \Gamma ; \mathbb{C}\right)$ induced from $(f, c) \rightarrow c$ is the same as the map $\mathrm{H}_{c}^{1}\left(H^{n} / \Gamma ; \mathbb{C}\right) \rightarrow \mathrm{H}^{1}\left(H^{n} / \Gamma ; \mathbb{C}\right)$. Its kernel can be identified with the $\Gamma$-invariant 
locally-constant functions on $\Omega$, modulo $\mathbb{C}$. This has dimension equal to the number of ends of $H^{n} / \Gamma$ minus one, as it should.

Choose $x \in \mathrm{H}_{c}^{1}\left(H^{n} / \Gamma ; \mathbb{C}\right)$. Define the locally-constant function $f: \Omega \rightarrow \mathbb{C}$ as in the proof of Lemma 3. As $\Lambda$ has measure zero, we can think of $f$ as a measurable function on $S^{n-1}$.

Proposition 6. $f$ lies in $\mathrm{L}^{p}\left(S^{n-1}\right)$ for all $p \in[1, \infty)$.

Proof. Let $K$ be the convex core of $H^{n} / \Gamma$ and let $\partial K$ be its boundary. Put $\widetilde{K}=\pi^{-1}(K)$, the convex hull of $\Lambda$, and put $\widetilde{\partial K}=\pi^{-1}(\partial K)$. As $\widetilde{K}$ is convex and $K$ is compact, it follows that $\widetilde{\partial K}$ is quasi-convex, meaning that there is an $R>0$ such that if $y_{1}, y_{2} \in \widetilde{\partial K}$ then the geodesic from $y_{1}$ to $y_{2}$, in $H^{n}$, lies in an $R$-neighborhood of $\partial K$. We take a ball model $B^{n}$ for $H^{n}$ such that $x_{0}=\pi(0)$ lies in $K$.

If $\Omega \subset S^{n-1}$ is connected then the result is trivial, so we assume that $\Omega$ has more than one connected component. Let $D$ be a connected component of $\Omega$. We first estimate the spherical volume of $D$. There is an end $e$ of $H^{n} / \Gamma$ such that if a curve $c$ in $H^{n}$ goes to $D$ then $\pi \circ c$ exits $e$. Let $\partial_{e} K$ be the connected component of $\partial K$ corresponding to $e$. Then there is a component $\widetilde{\partial_{D} K}$ of $\pi^{-1}\left(\partial_{e} K\right)$ such that $D$ retracts onto $\widetilde{\partial_{D} K}$ under the nearest-point retraction. Furthermore, the closure of $\widetilde{\partial_{D} K}$ in $\overline{B^{n}}$ separates $D$ from $K-\widetilde{\partial_{D} K}$. Let $r_{D}$ be the hyperbolic distance from 0 to $\widetilde{\partial_{D} K}$. Then $\widetilde{\partial_{D} K} \subset H^{n}-B_{r_{D}}(0)$. We are interested in what happens when $r_{D}$ is large. If $z_{1}, z_{2} \in \partial \bar{D}$ then the geodesic from $z_{1}$ to $z_{2}$ cannot enter $B_{r_{D}-R}(0)$, as this would violate the quasi-convexity of $\widetilde{\partial K}$. Quantitatively, this implies that the spherical distance from $z_{1}$ to $z_{2}$ cannot exceed $2 \sin ^{-1}\left(\frac{1}{\cosh \left(r_{D}-R\right)}\right)$. Thus $D$ lies within a spherical ball of radius $r_{0}=4 \sin ^{-1}\left(\frac{1}{\cosh \left(r_{D}-R\right)}\right)$. As the volume of this spherical ball is bounded above by a constant times $r_{0}^{n-1}$, we conclude that there is a constant $C>0$ such that $\operatorname{vol}(D) \leq C e^{-(n-1) r_{D}}$, uniformly in the choice of $D$.

The connected components of $\Omega$ are in one-to-one correspondence with the set $\pi_{1}(K, \partial K)$. Fix an end $e$ of $M$, with associated connected component $\partial_{e} K$ of $\partial K$. Take the ball model so that $x_{0} \in \partial_{e} K$. The connected components $D$ of $\Omega$ corresponding to $e$ form the preimage of $\partial_{e} K$ under the map $\pi_{1}(K, \partial K) \rightarrow \pi_{0}(\partial K)$. Given $D$, let $c(s), 0 \leq s \leq r_{D}$, be a normalized minimal geodesic from 0 to $\widetilde{\partial_{D} K}$. Consider a loop $L_{D}$ in $H^{n} / \Gamma$ which starts at $x_{0}$, follows $\pi \circ c$ to $\pi\left(c\left(r_{D}\right)\right) \in \partial_{e} K$ and then returns to $x_{0}$ by a length-minimizing path in $\partial_{e} K$. The length of $L_{D}$ will be bounded above by $r_{D}+\operatorname{diam}\left(\partial_{e} K\right)$. On the other hand, $L_{D}$ describes a class $\left[L_{D}\right] \in \pi_{1}\left(K, x_{0}\right)$. It follows that $d\left(0,\left[L_{D}\right] \cdot 0\right) \leq \operatorname{length}\left(L_{D}\right)$. Also, as $c$ is 
minimal from 0 to $c\left(r_{D}\right)$, we have $r_{D} \leq d\left(0,\left[L_{D}\right] \cdot 0\right)+\operatorname{diam}\left(\partial_{e} K\right)$. Thus

$$
d\left(0,\left[L_{D}\right] \cdot 0\right) \leq \operatorname{length}\left(L_{D}\right) \leq r_{D}+\operatorname{diam}\left(\partial_{e} K\right) \leq d\left(0,\left[L_{D}\right] \cdot 0\right)+2 \operatorname{diam}\left(\partial_{e} K\right) .
$$

In terms of the homotopy sequence

$$
\pi_{1}\left(K, x_{0}\right) \stackrel{\alpha}{\rightarrow} \pi_{1}(K, \partial K) \stackrel{\beta}{\rightarrow} \pi_{0}(\partial K)
$$

we have defined a map $s: \beta^{-1}\left(\partial_{e} K\right) \rightarrow \pi_{1}\left(K, x_{0}\right)$ which sends $D$ to $\left[L_{D}\right]$, with $\alpha \circ s=\operatorname{Id}$ on $\beta^{-1}\left(\partial_{e} K\right)$. Thus $s$ is injective. By the construction of $f$, there is a bound

$$
|f(D)| \leq A \text { length }\left(L_{D}\right)+B \leq A d\left(0,\left[L_{D}\right] \cdot 0\right)+B^{\prime}
$$

for $D \in \beta^{-1}\left(\partial_{e} K\right)$. Then

$$
\begin{aligned}
\sum_{D \in \beta^{-1}\left(\partial_{e} K\right)}|f(D)|^{p} \operatorname{vol}(D) \leq \sum_{D \in \beta^{-1}\left(\partial_{e} K\right)}\left(A d\left(0,\left[L_{D}\right] \cdot 0\right)+B^{\prime}\right)^{p} . \\
C e^{-(n-1)\left(d\left(0,\left[L_{D}\right] \cdot 0\right)-\operatorname{diam}\left(\partial_{e} K\right)\right)} .
\end{aligned}
$$

By [14], there is an $\epsilon>0$ such that

$$
\sum_{\gamma \in \Gamma} e^{-(n-1-\epsilon) d(0, \gamma \cdot 0)}<\infty
$$

It follows that $f$ is $\mathrm{L}^{p}$ on $\bigcup\left\{D \in \beta^{-1}\left(\partial_{e} K\right)\right\}$. Considering together the finite number of ends of $H^{n} / \Gamma$, the proposition follows.

Lemma 4. For $f \in L^{2}\left(S^{n-1}\right)$, let $\Phi_{0} f \in C^{\infty}\left(H^{n}\right)$ be its harmonic extension. For $1 \leq j \leq n$, let $x_{j}$ be the restriction to $S^{n-1}$ of the $j$-th coordinate function on $\mathbb{R}^{n}$. Then

$$
\left|\nabla\left(\Phi_{0} f\right)\right|^{2}(0)=(n-1)^{2} \sum_{j=1}^{n}\left|\frac{\int_{S^{n-1}} x_{j} f d v o l}{\operatorname{vol}\left(S^{n-1}\right)}\right|^{2} .
$$

Proof. Let $\left\{\beta_{i}\right\}_{i=1}^{\infty}$ be an orthonormal basis of $L^{2}\left(S^{n-1}\right)$ consisting of eigenvectors of $\triangle_{S^{n-1}}$ with eigenvalue $\left(k_{i}+1\right)\left(k_{i}+n-1\right), k_{i} \in \mathbb{Z} \cap[-1, \infty)$. Let $f=\sum_{i=1}^{\infty} a_{i} \beta_{i}$ be the Fourier expansion of $f$. Then from [6, p. 599],

$\left(\Phi_{0} f\right)(r, \theta)=\frac{\Gamma\left(\frac{n}{2}\right)}{\Gamma(n-1)} \sum_{i=1}^{\infty} a_{i} \frac{\Gamma\left(n+k_{i}\right)}{\Gamma\left(\frac{n}{2}+k_{i}+1\right)} r^{1+k_{i}} F\left(1-\frac{n}{2}, 1+k_{i} ; 1+\frac{n}{2}+k_{i} ; r^{2}\right) \beta_{i}(\theta)$.

It follows that

$$
\left|\nabla\left(\Phi_{0} f\right)\right|^{2}(0)=\frac{(n-1)^{2}}{n^{2}} \sum_{k_{i}=0}\left|a_{i}\right|^{2}\left(\left|\beta_{i}\right|^{2}+\left|\nabla_{S^{n-1}} \beta_{i}\right|^{2}\right) .
$$


We can take the $\beta_{i}$ 's with $k_{i}=0$ to be the functions $\left\{\left(\frac{n}{\operatorname{vol}\left(S^{n-1}\right)}\right)^{\frac{1}{2}} x_{j}\right\}_{j=1}^{n}$. In this case, one can verify that $\left|\beta_{i}\right|^{2}+\left|\nabla_{S^{n-1}} \beta_{i}\right|^{2}$ is constant on $S^{n-1}$. Its integral is

$$
\int_{S^{n-1}}\left(\left|\beta_{i}\right|^{2}+\left|\nabla_{S^{n-1}} \beta_{i}\right|^{2}\right) d \mathrm{vol}=\left\langle\beta_{i}, \beta_{i}\right\rangle+\left\langle\beta_{i}, \triangle_{S^{n-1}} \beta_{i}\right\rangle=1+(n-1)=n .
$$

Hence

$$
\left|\beta_{i}\right|^{2}+\left|\nabla_{S^{n-1}} \beta_{i}\right|^{2}=\frac{n}{\operatorname{vol}\left(S^{n-1}\right)}
$$

and so

$$
\begin{aligned}
\left|\nabla\left(\Phi_{0} f\right)\right|^{2}(0) & =\frac{(n-1)^{2}}{n \operatorname{vol}\left(S^{n-1}\right)} \sum_{k_{i}=0}\left|a_{i}\right|^{2} \\
& =\frac{(n-1)^{2}}{n \operatorname{vol}\left(S^{n-1}\right)} \sum_{j=1}^{n}\left|\int_{S^{n-1}}\left(\frac{n}{\operatorname{vol}\left(S^{n-1}\right)}\right)^{\frac{1}{2}} x_{j} f d \mathrm{vol}\right|^{2} \\
& =(n-1)^{2} \sum_{j=1}^{n}\left|\frac{\int_{S^{n-1}} x_{j} f d v o l}{\operatorname{vol}\left(S^{n-1}\right)}\right|^{2}
\end{aligned}
$$

The lemma follows.

Proposition 7. $d\left(\Phi_{0} f\right)$ is a $\Gamma$-invariant harmonic 1 -form on $H^{n}$. It descends to an $L^{2}$-harmonic 1-form on $H^{n} / \Gamma$.

Proof. As $f$ is $L^{2}, \Phi_{0} f$ is well-defined. As $\Phi_{0} f$ is harmonic, $\triangle_{1} d\left(\Phi_{0} f\right)=$ $d\left(\triangle_{0} \Phi_{0} f\right)=0$. Thus $d\left(\Phi_{0} f\right)$ is harmonic. Furthermore, for all $\gamma \in \Gamma$,

$$
d\left(\Phi_{0} f\right)-\gamma \cdot d\left(\Phi_{0} f\right)=d\left(\Phi_{0}(f-\gamma \cdot f)\right)=d\left(\Phi_{0} c_{\gamma}\right)=d c_{\gamma}=0 .
$$

Thus $d\left(\Phi_{0} f\right)$ is $\Gamma$-invariant. It remains to show that the descent of $d\left(\Phi_{0} f\right)$ to $H^{n} / \Gamma$ is $L^{2}$.

Let $m$ be a point in the connected component of $H^{n} / \Gamma-K$ corresponding to an end $e$. Take a ball model $B^{n}$ of $H^{n}$ with $\pi(0)=m$. Let $D$ be the connected component of $\Omega$ adjacent, in $\overline{B^{n}}$, to the connected component of $H^{n}-\widetilde{K}$ containing 0 . Changing $f$ by a constant, we may assume that $f$ vanishes on $D$. The method of proof of Proposition 6 implies that the $L^{1}$-norm of $f$, as seen in the visual sphere at $m$, is $O\left(e^{-(n-1) d(m, K)}\right)$ with respect to $m$. Then by Lemma 4 ,

$$
\left|\nabla\left(\Phi_{0} f\right)\right|^{2}(0)=O\left(e^{-2(n-1) d(m, K)}\right) .
$$

On the other hand, the volume of $\left\{m \in H^{n} / \Gamma: d(m, K) \in[j, j+1]\right\}$ is $O\left(e^{(n-1) j}\right)$. The proposition follows. 
Thus we have constructed $\operatorname{dim}\left(\mathrm{H}_{c}^{1}\left(H^{n} / \Gamma ; \mathbb{C}\right)\right)$ linearly-independent $L^{2}$-harmonic 1-forms on $H^{n} / \Gamma$.

Theorem 4.B. Let $\Gamma$ be a convex-cocompact group which is not cocompact. Let $\alpha$ be a nonzero $L^{2}$-harmonic 1 -form on $H^{n} / \Gamma$. Then $\Phi_{1}^{-1}\left(\pi^{*} \alpha\right)$ is Sobolev $\mathrm{H}^{-1}$ regular.

Proof. We know that $\pi^{*} \alpha=d\left(\Phi_{0} f\right)$ for some $f \in L^{2}\left(S^{n-1}\right)$ constructed as in Lemma 3. Then $\pi^{*} \alpha=\Phi_{1}(d f)$, with $d f$ being Sobolev $\mathrm{H}^{-1}$-regular.

\section{Surfaces}

Theorem 5.A. Let $\Gamma$ be a torsion-free uniform lattice in $\operatorname{Isom}^{+}\left(H^{2}\right)$, with $H^{2} / \Gamma$ a closed surface of genus $g$. Then

1. $\operatorname{dim}\left(\mathcal{A}_{0}^{\prime}\left(S^{1}\right)\right)^{\Gamma}=2 g$.

2. $\operatorname{dim}\left(\mathcal{D}_{0}^{\prime}\left(S^{1}\right)\right)^{\Gamma}=2 g$.

3. $\operatorname{dim}\left(\mathcal{D} Z\left(S^{1}\right) / \mathbb{C}\right)^{\Gamma}=2 g$.

4. $\operatorname{dim}\left(L^{2}\left(S^{1}\right) / \mathbb{C}\right)^{\Gamma}=0$.

Proof. The proof is similar to the proof of Theorem 2.A. If $F \in\left(\mathcal{A}_{0}^{\prime}\left(S^{1}\right)\right)^{\Gamma}$ then $d F$ is a $\Gamma$-invariant exact hyperform on $S^{1}$ and $\Phi_{1}(d F)$ is a $\Gamma$-invariant closed and coclosed 1-form on $H^{2}$. Thus $\Phi_{1}(d F)=\pi^{*} \alpha$ for a harmonic 1-form on $H^{2} / \Gamma$. In terms of the complex coordinate $z$ on $D^{2}$, we can write $\Phi_{1}(d F)=h_{1}(z) d z+h_{2}(\bar{z}) d \bar{z}$ where $h_{1}(z)$ and $h_{2}(z)$ are holomorphic functions. Let $k_{1}(z)$ and $k_{2}(z)$ satisfy $h_{i}(z)=k_{i}^{\prime \prime}(z)$ for $i \in\{1,2\}$. Then

$$
d\left(\Phi_{0} F\right)=\Phi_{1}(d F)=d\left(k_{1}^{\prime}(z)+k_{2}^{\prime}(\bar{z})\right),
$$

so $\Phi_{0} F=k_{1}^{\prime}(z)+k_{2}^{\prime}(\bar{z})+$ const. As $\alpha$ is bounded, $\Phi_{1}(d F)$ is uniformly bounded on $H^{2}$ and so

$$
\sup _{z \in D^{2}}\left(1-|z|^{2}\right)\left|k_{i}^{\prime \prime}(z)\right|<\infty
$$

That is, $k_{i}^{\prime}$ is an element of the Bloch space and so $k_{i}$ has a boundary value in the Zygmund functions $\mathcal{Z}\left[7\right.$, p. 282,442]. Thus $F(\theta)=k_{1}^{\prime}\left(e^{i \theta}\right)+k_{2}^{\prime}\left(e^{-i \theta}\right)+$ const., showing that $F$ has the required regularity.

Part (4) follows from the fact that $\Gamma$ acts ergodically on $S^{1}$.

Theorem 5.B. Let $\Gamma$ be a torsion-free nonuniform lattice in $\operatorname{Isom}^{+}\left(H^{2}\right)$, with $H^{2} / \Gamma$ the complement of $k$ points in a closed surface $S$ of genus $g$. Then 1. $\operatorname{dim}\left(\mathcal{A}_{0}^{\prime}\left(S^{1}\right)\right)^{\Gamma}=\infty$. 
2. $\operatorname{dim}\left(\mathcal{D}_{0}^{\prime}\left(S^{1}\right)\right)^{\Gamma}=\max (2 g, 2 g+2 k-2)$.

3. $\operatorname{dim}\left(H^{-\frac{1}{2}}\left(S^{1}\right) / \mathbb{C}\right)^{\Gamma}=2 g$.

4. $\operatorname{dim}\left(\mathcal{D} Z\left(S^{1}\right) / \mathbb{C}\right)^{\Gamma}=2 g$.

5. $\operatorname{dim}\left(L^{2}\left(S^{1}\right) / \mathbb{C}\right)^{\Gamma}=0$.

Proof. Sending $f \in\left(\mathcal{A}_{0}^{\prime}\left(S^{1}\right)\right)^{\Gamma}$ to $\Phi_{1}(d f)$, we see that $\left(\mathcal{A}_{0}^{\prime}\left(S^{1}\right)\right)^{\Gamma}$ is isomorphic to the space of closed and coclosed 1-forms on $H^{2} / \Gamma$. Let $p$ be a puncture point in $S$ and let $\mathbb{Z}$ be the subgroup of $\Gamma$ generated by a loop around $p$. Then the cusp of $H^{2} / \Gamma$ corresponding to $p$ embeds in $H^{2} / \mathbb{Z}$. We model the latter by the upper-half-plane quotiented by $z \rightarrow z+1$. Consider the pullback of $\Phi_{1}(d f)$ under the quotient map $H^{2} / \mathbb{Z} \rightarrow H^{2} / \Gamma$. As in [8], such a 1 -form on $H^{2} / \mathbb{Z}$ can be written as $h_{1}(z) d z+h_{2}(\bar{z}) d \bar{z}$, where $h_{i}(z)=h_{i}(z+1)$. Each $h_{i}$ has a Fourier expansion

$$
h_{i}(z)=\sum_{j \in \mathbb{Z}} c_{i, j} e^{2 \pi \sqrt{-1} j z}
$$

If $c_{1, j}=0$ for $j<-J$ then a change of variable $w=e^{2 \pi \sqrt{-1} z}$ gives

$$
h_{1}(z) d z=\sum_{j \geq-J} c_{1, j} w^{j-1} \frac{d w}{2 \pi \sqrt{-1}}
$$

and similarly for $h_{2}(\bar{z}) d \bar{z}$.

To each puncture point $p_{l} \in S, 1 \leq l \leq k$, assign an integer $J_{l}$ and let $i\left(-\sum_{l=1}^{k}\left(J_{l}+1\right) p_{l}\right)$ denote the space of holomorphic differentials on $S$ whose Laurent expansion around each $p_{l}$ has the form of the right-hand-side of (4.4) with $J=J_{l}$. By the Riemann-Roch theorem, $i(D) \geq g-1+\sum_{l=1}^{k}\left(J_{l}+1\right)$. Taking the numbers $\left\{J_{l}\right\}_{l=1}^{k}$ large, part (1) follows.

Part (2) was proven in [8]. For completeness, we repeat the argument. On the upper-half-plane, $\left|h_{1}(z) d z\right|=\left|h_{1}(x+i y)\right| y$. As $d(i, i y)=|\ln (y)|$, if $h_{1}(z) d z$ has slow growth as $y \rightarrow \infty$ then we must have $c_{1, j}=0$ for $j<0$. The space of such holomorphic differentials on $S$ has dimension $i\left(-\sum_{l=1}^{k} p_{l}\right)$. The Riemann-Roch theorem implies that $i\left(-\sum_{l=1}^{k} p_{l}\right)=\max (g+k, g+k-1)$. Part (2) follows.

Suppose that $f \in\left(\mathrm{H}^{-\frac{1}{2}}\left(S^{1}\right) / \mathbb{C}\right)^{\Gamma}$. Then $d f$ is $\mathrm{H}^{-\frac{3}{2}}$-regular. Considering $\Phi_{1}(d f)$, we know that on a cusp, $h_{1}(z)$ has an expansion (4.3) with $c_{1, j}=0$ for $j<0$. If $c_{1,0} \neq 0$ then as $y \rightarrow \infty, h_{1}(z) d z \sim c_{1,0} d z$. To analyze the singularity at a cusp point on $S^{1}$, we consider the 1 -form $c_{1,0} d z$ on the upper-half-plane and perform the reflection $z \rightarrow \frac{z}{|z|^{2}}$. On the boundary of the upper-half-plane, this restricts to $x \rightarrow \frac{1}{x}$ and so $c_{1,0} d x \rightarrow-c_{1,0} \frac{d x}{x^{2}}$. The point $i \infty$ gets mapped 
to 0 and so it is enough to look at the singularity of $-c_{1,0} \frac{d x}{x^{2}}$ near $x=0$. The Fourier transform of $\frac{1}{x^{2}}$ is proportionate to $|k|$. Hence $\frac{1}{x^{2}}$ lies in $\mathrm{H}^{s}$ if and only if $\int_{\mathbb{R}}\left(1+k^{2}\right)^{s}|k|^{2} d k<\infty$, i.e. if $s<-\frac{3}{2}$. This contradicts the assumption that $d f$ is $\mathrm{H}^{-\frac{3}{2}}$-regular. Thus $c_{1,0}=0$. Then $\Phi_{1}(d f)$ is bounded and as in the proof of Theorem 5.A, $f \in\left(\mathcal{D} Z\left(S^{1}\right) / \mathbb{C}\right)^{\Gamma}$. Furthermore, $h_{1}(z) d z$ extends smoothly over the puncture points to give a holomorphic differential on $S$. We conclude that both $\left(\mathrm{H}^{-\frac{1}{2}}\left(S^{1}\right) / \mathbb{C}\right)^{\Gamma}$ and $\left(\mathcal{D} Z\left(S^{1}\right) / \mathbb{C}\right)^{\Gamma}$ are isomorphic to two copies of the space of holomorphic differentials on $S$, the dimension of which is $g$. Parts (3) and (4) follow.

Finally, part (5) follows from the ergodicity of the $\Gamma$-action on $S^{1}$.

\section{3-Manifolds}

\subsection{Quasi-Fuchsian groups}

Let $S$ be a closed oriented surface of genus $g>1$. Let $\Gamma$ be a quasi-Fuchsian subgroup of $\operatorname{Isom}^{+}\left(H^{3}\right)$ which is isomorphic to $\pi_{1}(S)$. Then $H^{3} / \Gamma$ is diffeomorphic to $\mathbb{R} \times S$ and $H_{c}^{1}\left(H^{3} / \Gamma ; \mathbb{C}\right)=\mathbb{C}$. (In terms of the projection $p: \mathbb{R} \times S \rightarrow \mathbb{R}$, a proper map, one has $\mathrm{H}_{c}^{1}\left(H^{3} / \Gamma ; \mathbb{C}\right)=p^{*}\left(\mathrm{H}_{c}^{1}(\mathbb{R} ; \mathbb{C})\right)$ ). Thus there is a nonzero $L^{2}$-harmonic 1-form $\alpha$ on $H^{3} / \Gamma$.

By Corollary 2 and Proposition $3, \Phi_{1}^{-1}\left(\pi^{*} \alpha\right)$ is a $\Gamma$-invariant exact 1-current supported on the limit set $\Lambda \subset S^{2}$. The domain of discontinuity $\Omega \subset S^{2}$ is the union of two 2-disks $D_{+}$and $D_{-}$, with $D_{+} / \Gamma$ and $D_{-} / \Gamma$ homeomorphic to $S$. Let $\chi_{D_{+}} \in L^{2}\left(S^{2}\right)$ be the characteristic function of $D_{+}$. By Proposition $7, \Phi_{1}^{-1}\left(\pi^{*} \alpha\right)$ is proportionate to the exact 1-current $d \chi_{D_{+}}$on $S^{2}$.

In order to write $d \chi_{D_{+}}$more directly on $\Lambda$, we follow the general scheme of [5, Section IV.3. $\gamma$ ]. Let $Z: D^{2} \rightarrow D_{+}$be a uniformization of $D_{+}$. By Carathéodory's theorem, $Z$ extends to a continuous homeomorphism $\bar{Z}: \overline{D^{2}} \rightarrow \overline{D_{+}}$. The restriction of $\bar{Z}$ to $\partial \overline{D^{2}}$ gives a homeomorphism $\partial \bar{Z}: S^{1} \rightarrow \Lambda$.

From a general construction [5, Theorem 2, p. 208], the 1-current $d \chi_{D_{+}}$defines a cyclic 1-cocycle $\tau$ on the algebra $C^{1}\left(S^{2}\right)$ by

$$
\tau\left(F^{0}, F^{1}\right)=\int_{S^{2}} d \chi_{D_{+}} \wedge F^{0} d F^{1}
$$

Lemma 1. The function space $\mathrm{H}^{\frac{1}{2}}\left(S^{1}\right) \cap L^{\infty}\left(S^{1}\right)$ is a Banach algebra with the norm

$$
\|f\|=\left(\int_{\mathbb{R}^{+}} \int_{S^{1}} \frac{|f(\theta+h)-f(\theta)|^{2}}{h^{2}} d \theta d h\right)^{\frac{1}{2}}+\|f\|_{\infty} .
$$


Given $f^{0}, f^{1} \in \mathrm{H}^{\frac{1}{2}}\left(S^{1}\right) \cap L^{\infty}\left(S^{1}\right)$, let

$$
f^{i}(\theta)=\sum_{j \in \mathbb{Z}} c_{j}^{i} e^{\sqrt{-1} j \theta}
$$

be the Fourier expansion. Define a bilinear function

$$
\bar{\tau}:\left(\mathrm{H}^{\frac{1}{2}}\left(S^{1}\right) \cap L^{\infty}\left(S^{1}\right)\right) \times\left(\mathrm{H}^{\frac{1}{2}}\left(S^{1}\right) \cap L^{\infty}\left(S^{1}\right)\right) \rightarrow \mathbb{C}
$$

by

$$
\bar{\tau}\left(f^{0}, f^{1}\right)=-2 \pi i \sum_{j \in \mathbb{Z}} j c_{j}^{0} c_{-j}^{1} .
$$

Then $\bar{\tau}$ is a continuous cyclic 1 -cocycle on $\mathrm{H}^{\frac{1}{2}}\left(S^{1}\right) \cap L^{\infty}\left(S^{1}\right)$.

Proof. It is straightforward to check that $\mathrm{H}^{\frac{1}{2}}\left(S^{1}\right) \cap L^{\infty}\left(S^{1}\right)$ is a Banach algebra with the given norm. It is also easy to check that $\bar{\tau}$ is continuous. If $f^{0}, f^{1} \in$ $C^{\infty}\left(S^{1}\right)$ then

$$
\bar{\tau}\left(f^{0}, f^{1}\right)=\int_{S^{1}} f^{0} d f^{1} .
$$

As in $[5$, p. 182], put

$$
(b \bar{\tau})\left(f^{0}, f^{1}, f^{2}\right)=\bar{\tau}\left(f^{0} f^{1}, f^{2}\right)-\bar{\tau}\left(f^{0}, f^{1} f^{2}\right)+\bar{\tau}\left(f^{2} f^{0}, f^{1}\right) .
$$

If $f^{0}, f^{1}, f^{2} \in C^{\infty}\left(S^{1}\right)$ then $(b \bar{\tau})\left(f^{0}, f^{1}, f^{2}\right)=0$. As $C^{\infty}\left(S^{1}\right)$ is dense in $\mathrm{H}^{\frac{1}{2}}\left(S^{1}\right) \cap$ $L^{\infty}\left(S^{1}\right)$ and $b \bar{\tau}$ is continuous in its arguments, it follows that $b \bar{\tau}=0$.

Theorem 6. Given $F^{0}, F^{1} \in C^{1}\left(S^{2}\right)$, put $f^{i}=(\partial \bar{Z})^{*} F^{i}, i \in\{1,2\}$. Then $f^{i} \in \mathrm{H}^{\frac{1}{2}}\left(S^{1}\right) \cap L^{\infty}\left(S^{1}\right)$ and

$$
\tau\left(F^{0}, F^{1}\right)=-\bar{\tau}\left(f^{0}, f^{1}\right)
$$

Proof. Consider $S^{2}$ as $\mathbb{C} \cup \infty$ with $\infty \in D_{-}$. For $r \in(0,1)$, let $i_{r}: S^{1} \rightarrow D^{2}$ be the embedding of $S^{1}$ as the circle of radius $r$ around $0 \in D^{2}$. Thinking of $Z$ as a map from $D^{2}$ to $\mathbb{C}$, let

$$
Z(z)=\sum_{k=0}^{\infty} c_{k} z^{k}
$$

be its Taylor's series. Then

$$
\frac{i}{2} \int_{B_{r}(0)} d Z \wedge d Z^{*}=\frac{i}{2} \int_{S^{1}} i_{r}^{*} Z d\left(i_{r}^{*} Z^{*}\right)=\pi \sum_{k=0}^{\infty} k r^{2 k}\left|c_{k}\right|^{2} .
$$


As $Z$ is univalent,

$$
\frac{i}{2} \int_{D^{2}} d Z \wedge d Z^{*}=\operatorname{area}\left(Z\left(D^{2}\right)\right)<\infty
$$

It follows that

$$
\lim _{r \rightarrow 1} i_{r}^{*} Z=\partial \bar{Z}
$$

in $\mathrm{H}^{\frac{1}{2}}\left(S^{1}\right) \cap L^{\infty}\left(S^{1}\right)$. Then $f^{i} \in \mathrm{H}^{\frac{1}{2}}\left(S^{1}\right) \cap L^{\infty}\left(S^{1}\right)$.

We have

$$
\begin{aligned}
\tau\left(F^{0}, F^{1}\right) & =\int_{S^{2}} d \chi_{D^{+}} \wedge F^{0} d F^{1} \\
& =-\int_{S^{2}} \chi_{D^{+}} d F^{0} \wedge d F^{1} \\
& =-\int_{D^{+}} d F^{0} \wedge d F^{1} \\
& =-\int_{D^{2}} d\left(Z^{*} F^{0}\right) \wedge d\left(Z^{*} F^{1}\right) .
\end{aligned}
$$

Then

$$
\begin{aligned}
\tau\left(F^{0}, F^{1}\right) & =\lim _{r \rightarrow 1}-\int_{B_{r}(0)} d\left(Z^{*} F^{0}\right) \wedge d\left(Z^{*} F^{1}\right) \\
& =\lim _{r \rightarrow 1}-\int_{S^{1}} i_{r}^{*} Z^{*} F^{0} \wedge d\left(i_{r}^{*} Z^{*} F^{1}\right) \\
& =\lim _{r \rightarrow 1}-\bar{\tau}\left(i_{r}^{*} Z^{*} F^{0}, i_{r}^{*} Z^{*} F^{1}\right) .
\end{aligned}
$$

From (5.12),

$$
\lim _{r \rightarrow 1} i_{r}^{*} Z^{*} F^{i}=f^{i}
$$

in $\mathrm{H}^{\frac{1}{2}}\left(S^{1}\right) \cap L^{\infty}\left(S^{1}\right)$. The theorem follows.

Example. Let $\Sigma$ be a closed oriented surface of genus $g>2$, let $\phi \in \operatorname{Diff}(\Sigma)$ be an orientation-preserving pseudo-Anosov diffeomorphism and let $M$ be the mapping torus of $\phi$. Then $M$ is a 3-manifold which fibers over the circle and admits a hyperbolic structure $[16,13]$. Let $\widehat{M}=H^{3} / \Gamma$ be the corresponding cyclic cover of $M$, with the pullback hyperbolic metric. The group $\Gamma$ is isomorphic to $\pi_{1}(\Sigma)$. From [10, Proposition 9], $\widehat{M}$ has no nonzero $L^{2}$-harmonic 1-forms. This contrasts with the quasi-Fuchsian case. 


\subsection{Covering spaces}

If $M$ is a closed 3-manifold then $M$ has nontrivial $L^{2}$-harmonic 1-forms if and only if $b_{1}(M)>0$. There are many examples of hyperbolic manifolds 3-manifolds $M$ with $b_{1}(M)>0$, such as those which fiber over a circle. It is less obvious that there are infinite normal covers $\widehat{M}=H^{3} / \Gamma$ of closed hyperbolic 3-manifolds such that $\widehat{M}$ has nonzero $L^{2}$-harmonic 1 -forms. We give some examples. The limit sets will be all of $S^{2}$.

Let $M$ be a closed oriented hyperbolic 3-manifold with a surjective homomorphism $\alpha: \pi_{1}(M) \rightarrow F_{r}$ onto a free group with $r>1$ generators. Let $\widehat{M}=H^{3} / \Gamma$ be the corresponding cover with $\Gamma \cong \operatorname{Ker}(\alpha)$. The space of ends of $\widehat{M}$ is a Cantor set. As $F_{r}$ is nonamenable, Proposition 5 applies to show that $\widehat{M}$ has an infinitedimensional space of $L^{2}$-harmonic 1-forms. Thus for all $\epsilon>0,\left(\mathrm{H}^{-\epsilon}\left(S^{2}\right) / \mathbb{C}\right)^{\Gamma}$ is infinite-dimensional.

For another example, let $\Sigma$ be a closed oriented surface of genus $g>2$. Let $\rho$ be a nonzero element of $\mathrm{H}^{1}(\Sigma ; \mathbb{Z})=\mathbb{Z}^{2 g}$. Let $\widehat{\Sigma}$ be the cyclic cover of $\Sigma$ coming from the homomorphism $\pi_{1}(\Sigma) \rightarrow \mathrm{H}_{1}(\Sigma ; \mathbb{Z}) \stackrel{\rho}{\rightarrow} \mathbb{Z}$. It is an infinite-genus surface.

Let $\phi$ be an orientation-preserving pseudo-Anosov diffeomorphism of $\Sigma$ which acts trivially on $\mathrm{H}^{1}(\Sigma ; \mathbb{Z})$; it is a surprising fact that such diffeomorphisms exist [17]. It lifts to a diffeomorphism $\widehat{\phi}$ of $\widehat{\Sigma}$. Let $M$ be the mapping torus of $\phi$, with its hyperbolic metric. It follows from the Wang sequence that $\mathrm{H}^{1}(M ; \mathbb{Z})=\mathbb{Z}^{2 g} \oplus \mathbb{Z}$. Let $\widehat{M}=H^{3} / \Gamma$ be the cyclic covering of $M$ coming from $\rho \oplus 0 \in \mathrm{H}^{1}(M ; \mathbb{Z})$. Equivalently, $\widehat{M}$ is the mapping torus of $\widehat{\phi}$.

Given $e^{i \theta} \in U(1)$, let $\rho_{\theta}: \mathbb{Z} \rightarrow U(1)$ be the representation $\rho_{\theta}(n)=e^{i n \theta}$. Let $E_{\theta}$ be the flat unitary line bundle on $\Sigma$ coming from the representation $\pi_{1}(\Sigma) \rightarrow$ $\mathrm{H}_{1}(\Sigma ; \mathbb{Z}) \stackrel{\rho}{\rightarrow} \mathbb{Z} \stackrel{\rho_{\theta}}{\rightarrow} U(1)$. Let $F_{\theta}$ be the flat unitary line bundle on $M$ coming from the representation $\pi_{1}(M) \rightarrow \mathrm{H}_{1}(M ; \mathbb{Z}) \stackrel{\rho \oplus 0}{\rightarrow} \mathbb{Z} \stackrel{\rho_{\theta}}{\rightarrow} U(1)$; it is the mapping torus for the action of $\phi$ on $E_{\theta}$. As in [10, Section 4], it follows from Fourier analysis that $\widehat{M}$ has a nonzero $L^{2}$-harmonic 1 -form if and only if $\mathrm{H}^{1}\left(M ; F_{\theta}\right) \neq 0$ for all $\theta$. Furthermore, because of the $\mathbb{Z}$-action on $\widehat{M}$, if there is one nonzero $L^{2}$-harmonic 1-form then there is an infinite-dimensional space.

From the Euler characteristic identity and Poincaré duality,

$$
2-2 g=2 \operatorname{dim} \mathrm{H}^{0}\left(\Sigma ; E_{\theta}\right)-\operatorname{dim} \mathrm{H}^{1}\left(\Sigma ; E_{\theta}\right) .
$$

As $\operatorname{dim} \mathrm{H}^{0}\left(\Sigma ; E_{\theta}\right) \leq 1$, it follows that

$$
\operatorname{dim} \mathrm{H}^{1}\left(\Sigma ; E_{\theta}\right)=2 g-2\left(1-\operatorname{dim} \mathrm{H}^{0}\left(\Sigma ; E_{\theta}\right)\right)>0 .
$$

From the Wang sequence,

$$
\mathrm{H}^{1}\left(M ; F_{\theta}\right) \cong \mathrm{H}^{0}\left(\Sigma ; E_{\theta}\right) \oplus \mathrm{H}^{1}\left(\Sigma ; E_{\theta}\right) \neq 0 .
$$


Thus $\widehat{M}$ has nonzero $L^{2}$-harmonic 1-forms and for all $\epsilon>0,\left(\mathrm{H}^{-\epsilon}\left(S^{2}\right) / \mathbb{C}\right)^{\Gamma}$ is infinite-dimensional. The $L^{2}$-harmonic 1-forms on $\widehat{M}$ arise from the fact that $\operatorname{Im}\left(\mathrm{H}_{c}^{1}(\widehat{M} ; \mathbb{C}) \rightarrow \mathrm{H}^{1}(\widehat{M} ; \mathbb{C})\right)$ is nonzero.

\section{Acknowledgments}

I thank Dick Canary and Curt McMullen for helpful discussions. I thank Curt for asking questions that led to this work. I thank the IHES for its hospitality while some of the research was done.

\section{References}

[1] M. Abramowitz and I. Stegun, Handbook of Mathematical Functions, National Bureau of Standards, Washington 1964.

[2] R. Benedetti and C. Petronio, Lectures on Hyperbolic Geometry, Springer-Verlag, New York 1992.

[3] F. Bonahon, Bouts des Variétés Hyperboliques de Dimension 3, Ann. of Math. 124 (1986) 71-158.

[4] J. Cheeger, M. Gromov and M. Taylor, Finite Propagation Speed, Kernel Estimates for Functions of the Laplace Operator and the Geometry of Complete Riemannian Manifolds, J. Diff. Geom. 17 (1982), 15-53.

[5] A. Connes, Noncommutative Differential Geometry, Academic Press, San Diego 1994.

[6] P.-Y. Gaillard, Transformation de Poisson de Formes Differentielles. Le Cas de l'Espace Hyperbolique, Comm. Math. Helv. 61 (1986), 581-616.

[7] J. Garnett, Bounded Analytic Functions, Academic Press, New York 1981.

[8] A. Haefliger and L. Banghe, Currents on a Circle Invariant by a Fuchsian Group. In: Geometric Dynamics, Springer LNM 1007, ed. J. Palis, pp. 369-378. Springer-Verlag, New York 1983.

[9] J. Lott, The Zero-in-the-Spectrum Problem, L'Enseignement Math. 42 (1996), 341-376.

[10] J. Lott, $L^{2}$-Cohomology of Geometrically Infinite Hyperbolic 3-Manifolds, Geom. and Funct. Anal. 7 (1997), 81-119.

[11] Y. Luke, Integrals of Bessel Functions, McGraw-Hill, New York 1962.

[12] R. Mazzeo and R. Phillips, Hodge Theory on Hyperbolic Manifolds, Duke Math. J. 60 (1990), 509-559.

[13] J.-P. Otal, Le Théorème d'Hyperbolisation pour les Variétés Fibrées de Dimension 3, Astèrisque 235 (1996).

[14] D. Sullivan, The Density at Infinity of a Discrete Group of Hyperbolic Motions, Publ. IHES 50 (1979), 171-202.

[15] D. Sullivan, Related Aspects of Positivity in Riemannian Geometry, J. of Diff. Geom. 25 (1987), 327-351.

[16] W. Thurston, Hyperbolic Structures on 3-Manifolds II: Surface Groups and Manifolds which Fiber over the Circle, Version 2.0, preprint (1986).

[17] W. Thurston, On the Geometry and Dynamics of Diffeomorphisms of Surfaces, Bull. AMS 19 (1988), 417-431. 
John Lott

Department of Mathematics

University of Michigan

Ann Arbor, MI 48109-1109

USA

e-mail: lott@math.lsa.umich.edu

(Received: January 18, 2000) 